\title{
Phase Equilibria of Quasi-Ternary Systems Consisting of Multi- component Polymers in a Binary Solvent Mixture V. Effects of Concentration Dependence of Thermodynamic Interaction Parameters
}

\author{
Shigenobu MATSUDA \\ Fundamental Research Laboratory of Fibers and Fiber-Forming Polymers, \\ Asahi Chemical Industry Co., Ltd., \\ 11-7, Hacchonawate, Takatsuki, Osaka 569, Japan
}

(Received May 15, 1986)

\begin{abstract}
The concentration dependences of three $\chi$ parameters (thermodynamic interaction parameters of solvent $1-2$, solvent 1-polymer, and solvent 2-polymer; $\chi_{12}, \chi_{13}$, and $\chi_{23}$, respectively) were studied for developing a theory of the two-phase equilibrium of a quasi-ternary system which consists of multicomponent polymers and a binary solvent mixture. $\chi_{12}, \chi_{13}$, and $\chi_{23}$
\end{abstract} were defined by

$$
\begin{aligned}
& \chi_{12}=\chi_{12}^{0}\left(1+\sum_{s=1}^{n_{s}} p_{12, s} v_{p}^{s}\right) \\
& \chi_{13}=\chi_{13}^{0}\left(1+\sum_{q=1}^{n_{q}} p_{13, q} v_{p}^{q}\right) \\
& \chi_{23}=\chi_{23}^{0}\left(1+\sum_{r=1}^{n_{r}} p_{23, r} v_{p}^{r}\right)
\end{aligned}
$$

where $\chi_{12}^{0}, \chi_{13}^{0}$, and $\chi_{23}^{0}$ are the parameters independent of the total polymer concentration $v_{p}$ and are dependent on the temperature only, and $p_{12,1}, p_{13,1}$, and $p_{23,1}$ are the concentration dependent parameters. Computer experiments on solutions of an original polymer with the Schulz-Zimm type molecular weight distribution (the ratio of weight- to number-average molar volume of the polymer to the solvents $X_{w}^{0} / X_{n}^{0}=2.0$ and $X_{w}^{0}=300$ ) were carried out to study the effects of concentration dependent parameters on the two phase equilibrium characteristics. Partition coefficient $\sigma$, phase volume ratio $R$, and polymer volume fraction in polymer rich phase $v_{p(2)}$ increase with an increase in $p_{13,1}$ and $p_{23,1}$ and yield maximum near $p_{12,1}=-0.2$. The effects of the concentration dependence of $\chi$-parameter on $\sigma, R$, and $v_{p(2)}$ decrease in the following order: $p_{23,1}>p_{13,1}>p_{12,1}$.

KEY WORDS Concentration Dependent Parameter / Thermodynamic Interaction Parameter / Quasi-Ternary System / Binary Solvent Mixture / Partition Coefficient / Phase Volume Ratio /

The phase separation phenomena of monodisperse polymer/solvent/nonsolvent systems had been studied by Flory, ${ }^{1}$ Scott, ${ }^{2,3}$ Tompa, ${ }^{4}$ Nakagaki and Sunada, ${ }^{5}$ Krigbaum and Carpenter ${ }^{6}$ and Shu and Liou, ${ }^{7}$ but even for these relatively simple cases, two-phase equilibrium calculation had been based on crude assumptions: (1) a solvent mixture could be approximated as a "single solvent" (Flory, ${ }^{1}$ Scott $^{2,3}$ ), (2) polymer molecular weight was infinite (Scott, ${ }^{2,3}$ NakagakiSunada $a^{5}$ ), (3) among three thermodynamic interaction parameters $\chi_{12}, \chi_{13}$, and $\chi_{23}$ (originally defined in the Flory-Huggins theory: ${ }^{8}$ 
1 , solvent $1 ; 2$, solvent $2 ; 3$, polymer), the relationships that $\chi_{12}=\chi_{13}$ and $\chi_{23}=0$ held (Tompa, ${ }^{4}$ Nakagaki-Sunada ${ }^{5}$ and (4) polymer did not exist in the polymer-lean phase (Krigbaum-Carpenter, ${ }^{6}$ Shu-Liou ${ }^{7}$ ). Very recently ${ }^{9-11}$, we proposed a rigorous theory of phase equilibrium for a quasi-ternary system consisting of multicomponent polymers, solvent 1 (good solvent) and solvent 2 (poor solvent), where $\chi_{12}, \chi_{13}$, and $\chi_{23}$ were assumed to be concentration independent. Based on this theory, we carried out systematically a series of computer experiments on the quasi-ternary system to study the effects of (a) three thermodynamic interaction parameters, ${ }^{9}$ (b) the relative amount $\rho_{p}$ of polymer partitioned in a polymer rich phase and the initial concentration $v_{p}^{0},{ }^{10}$ and (c) the average molecular weight and molecular weight distribution (MWD) of the original polymer, ${ }^{11}$ on the phase separation characteristics, and compared the results with those of quasibinary systems consisting of multicomponent polymers and a single solvent. Aminavhabi and Munk, ${ }^{12}$ Altena and Smolders ${ }^{13}$ showed that the magnitude of $\chi_{12}$ and its concentration dependence influence thermodynamic properties, such as phase separation characteristics, of monodisperse polymer in binary solvent system. In this communication we have generalized the theory for phase equilibrium of quasi-ternary system studied in the previous papers $^{9-11}$ to the case where the three $\chi$ parameters (not only $\chi_{12}$ but also $\chi_{13}$ and $\chi_{23}$ ) depends significantly on the concentration, and explored the effects of these concentration dependence on the two-phase equilibrium characteristics.

\section{THEORETICAL BACKGROUND}

On the basis of Flory-Huggins theory, ${ }^{8}$ Kamide et al. $^{9-11}$ proposed a theory of quasiternary system, assuming that $\chi_{12}, \chi_{13}$, and $\chi_{23}$ are independent of the polymer molecular weight and concentration. Chemical potentials of solvent 1,2 , and $X_{i}$-mer $\left(\Delta \mu_{1}, \Delta \mu_{2}\right.$, and $\Delta \mu_{X i}$, respectively) were given by following equations.

$$
\begin{aligned}
\Delta \mu_{1}= & \tilde{R} T\left\{\ln v_{1}+\left(1-\frac{1}{X_{n}}\right) v_{p}\right. \\
& \left.+\chi_{12} v_{2}\left(1-v_{1}\right)+\chi_{13} v_{p}\left(1-v_{1}\right)-\chi_{23} v_{2} v_{p}\right\}
\end{aligned}
$$

$$
\begin{aligned}
\Delta \mu_{2}= & \tilde{R} T\left\{\ln v_{2}+\left(1-\frac{1}{X_{n}}\right) v_{p}\right. \\
& \left.+\chi_{12} v_{1}\left(1-v_{2}\right)+\chi_{23} v_{p}\left(1-v_{2}\right)-\chi_{13} v_{1} v_{p}\right\}
\end{aligned}
$$

$$
\begin{aligned}
& \Delta \mu_{X_{i}}= \tilde{R} T\left[\ln v_{X_{i}}-\left(X_{i}-1\right)+X_{i}\left(1-\frac{1}{X_{n}}\right) v_{p}\right. \\
&+X_{i}\left\{\chi_{13} v_{1}\left(1-v_{p}\right)+\chi_{23} v_{2}\left(1-v_{p}\right)\right. \\
&\left.\left.-\chi_{12} v_{1} v_{2}\right\}\right] \quad \\
&(i=1, \cdots, m)
\end{aligned}
$$

where $X_{i}$ is the molar volume ratio of the $i$ th polymer to solvent 1 or $2, v_{1}, v_{2}$, and $v_{X_{i}}$, the volume fraction of solvent 1,2 , and $X_{i}$-mer, respectively, $v_{p}$, the total polymer volume fraction $\left(\equiv \sum_{i=1}^{m} v_{X_{i}}\right), \quad \tilde{R}$ and $T, \quad$ the gas constant and Kelvin temperature, respectively. $m$ is the total number of polymer component, all belonging to the same chemical homologue. We assume that (a) the molar volume of solvent 1 is the same as that of solvent 2 , (b) solvent 1,2 , and polymer are volumetrically additive, and (c) the densities of solvent 1, 2, and polymer are the same. ${ }^{9}$ These assumptions do not limit the validity of the theory. For the quasi-binary system consisting of multicomponent polymers dissolved in a single solvent, we considered the concentration dependence of $\chi$ parameter, as follows. ${ }^{14}$

$$
\chi=\chi_{0}\left(1+\sum_{j=1}^{n} p_{j} v_{p}^{j}\right)
$$


Here, $\chi_{0}$ is the temperature dependent factor and $p_{j}(j=1, \cdots, n)$ are the concentration dependent parameters. From the analogy to the quasi-binary system, concentration dependence of $\chi_{12}, \chi_{13}$, and $\chi_{23}$ for the quasiternary system should be written as

$$
\begin{aligned}
\chi_{12}= & \chi_{12}^{0}\left(1+\sum_{s=1}^{n_{s}} p_{12, s} v_{p}^{s}\right) \\
& \times\left\{1+\sum_{t=1}^{n_{t}}\left(p_{1, t} v_{1}^{t}+p_{2, t} v_{2}^{t}\right)\right\} \\
& \chi_{13}=\chi_{13}^{0}\left(1+\sum_{q=1}^{n_{q}} p_{13, q} v_{p}^{q}\right) \\
& \chi_{23}=\chi_{23}^{0}\left(1+\sum_{r=1}^{n_{r}} p_{23, r} v_{p}^{r}\right)
\end{aligned}
$$

where $\chi_{12}^{0}, \chi_{13}^{0}$, and $\chi_{23}^{0}$ are parameters independent of the concentration and the degree of polymerization $\left(\sim X_{i}\right)$ and are dependent on temperature only. $p_{12, s}, p_{13, q}$, and $p_{23, r}$ are independent of $X_{i}$. and temperature. $p_{1, t}$ and $p_{2, t}$ are the solvent composition dependent parameters. Here, we neglected the theoretical and experimental possibility of the dependence of $\chi_{12}$ parameters on the composition of binary solvent mixture.

$$
\chi_{12}=\chi_{12}^{0}\left(1+\sum_{s=1}^{n_{s}} p_{12, s} v_{p}^{s}\right)
$$

Gibbs's free energy of mixing of these solvents and polymer, $\Delta G$ can be devided into four parts; Gibbs free energy of ideal solution, $\Delta G^{i d}$, excess free energy of solvent $1-2$, $\Delta G_{12}^{E}$, excess free energy of solvent 1polymer, $\Delta G_{13}^{E}$, and excess free energy of solvent 2-polymer, $\Delta G_{23}^{E}$ :

$$
\Delta G=\Delta G^{i d}+\Delta G_{12}^{E}+\Delta G_{13}^{E}+\Delta G_{23}^{E}
$$

Using eq 4 , the excess chemical potential of solvent $\partial \Delta G^{E} / \partial N_{0}$ for quasi-binary system becomes, ${ }^{14}$

$$
\frac{\partial \Delta G^{E}}{\partial N_{0}}=\tilde{R} T \chi_{0}\left(1+\sum_{j=1}^{n} p_{j} v_{p}^{j}\right) v_{p}^{2}
$$

where $N_{0}$ is the number of solvent molecules.
In the same way as for quasi-binary system, eq 6 and 7 are combined with the original equations of the exess chemical potential of solvents 1 and $2\left(\partial \Delta G_{13}^{E} / \partial N_{1}\right.$ and $\left.\partial \Delta G_{23}^{E} / \partial N_{2}\right)$, to give

$$
\begin{aligned}
& \frac{\partial \Delta G_{13}^{E}}{\partial N_{1}}=\tilde{R} T \chi_{13}^{0}\left(1+\sum_{q=1}^{n_{q}} p_{13, q} v_{p}^{q}\right) v_{p}\left(1-v_{1}\right) \\
& \frac{\partial G_{23}^{E}}{\partial N_{2}}=\tilde{R} T \chi_{23}^{0}\left(1+\sum_{r=1}^{n_{r}} p_{23, r} v_{p}^{r}\right) v_{p}\left(1-v_{2}\right)
\end{aligned}
$$

Here $N_{1}$ and $N_{2}$ are number of solvent 1 and 2 , respectively. Equation $5^{\prime}$ was directly put into an equation of $\Delta G_{12}^{E}$ (eq 12) in order to satisfy the symmetry with respect to the exchange of solvents 1 and 2 ,

$$
\begin{aligned}
\Delta G_{12}^{E} & \equiv \tilde{R} T L \chi_{12} v_{1} v_{2} \\
& =\tilde{R} T L \chi_{12}^{0}\left(1+\sum_{s=1}^{n_{s}} p_{12, s} v_{p}^{s}\right) v_{1} v_{2}
\end{aligned}
$$

where $L=N_{1}+N_{2}+\sum X_{i} N_{X_{i}}$ and $N_{X_{i}}$ is the number of $X_{i}$-mer. Combining eq $8,10-12$, we can determine $\Delta G$ of the quasi-ternary system and derivate $\Delta \mu_{1}, \Delta \mu_{2}$, and $\Delta \mu_{X_{i}}(i=1$, $\cdots, m$ ), respectively (see Appendix A), as

$$
\begin{aligned}
& \Delta \mu_{1}=\tilde{R} T\left[\ln v_{1}-\left(1-\frac{1}{X_{n}}\right) v_{p}\right. \\
& +\chi_{12}^{0}\left\{v_{2}\left(1-v_{1}\right)+\sum_{s=1}^{n_{s}} p_{12, s} v_{2} v_{p}^{s}\left(1-(s+1) v_{1}\right)\right\} \\
& +\chi_{13}^{0} v_{p}\left(1-v_{1}\right)\left\{1+\sum_{q=1}^{n_{q}} p_{13, q} v_{p}^{q}\right\} \\
& -\chi_{23}^{0} v_{2} v_{p}\left\{1+\sum_{r=1}^{n_{r}} p_{23, r} v_{p}^{r}+\sum_{r=1}^{n_{r}} p_{23, r}\left(\frac{r}{r+1}\right)\right. \\
& \left.\left.\times \frac{v_{p}^{r}}{\left(1-v_{2}\right)^{r+1}}\left(\frac{1-\left(v_{1}+v_{p}\right)^{r+1}}{v_{2}}\right)\right\}\right] \\
& \Delta \mu_{2}=\tilde{R} T\left[\ln v_{2}-\left(1-\frac{1}{X_{n}}\right) v_{p}\right. \\
& +\chi_{12}^{0}\left\{v_{1}\left(1-v_{2}\right)+\sum_{s=1}^{n_{s}} p_{12, s} v_{1} v_{p}^{s}\left(1-(s+1) v_{2}\right)\right\}
\end{aligned}
$$


S. MAtSuda

$$
\begin{aligned}
& +\chi_{23}^{0} v_{p}\left(1-v_{2}\right)\left\{1+\sum_{r=1}^{n_{r}} p_{23, r} v_{p}^{r}\right\}-\chi_{13}^{0} v_{1} v_{p} \\
& \times\left\{1+\sum_{q=1}^{n_{q}} p_{13, q} v_{p}^{q}+\sum_{q=1}^{n_{q}} p_{13, q}\left(\frac{q}{q+1}\right)\right. \\
& \left.\left.\times \frac{v_{p}^{q}}{\left(1-v_{1}\right)^{q+1}}\left(\frac{1-\left(v_{2}+v_{p}\right)^{q+1}}{v_{1}}\right)\right\}\right] \\
& \Delta \mu_{X_{i}}=R T\left[\ln v_{X_{i}}-\left(X_{i}-1\right)+X_{i}\left(1-\frac{1}{X_{n}}\right) v_{p}\right. \\
& -X_{i} \chi_{12}^{0} v_{1} v_{2}\left\{1-\sum_{s=1}^{n_{s}} p_{12, s} s v_{p}^{s-1}\right. \\
& \left.\times\left(1-\frac{s+1}{s} v_{p}\right)\right\} \\
& +X_{i} \chi_{13}^{0} v_{1}\left\{1-v_{p}\left(1+\sum_{q=1}^{n_{q}} p_{13, q} v_{p}^{q}\right)\right. \\
& +\sum_{q=1}^{n_{q}} p_{13, q}\left(1-\left(\frac{q}{q+1}\right) \frac{v_{p}}{1-v_{1}}\right)\left(\frac{v_{p}}{1-v_{1}}\right)^{q} \\
& \left.+\left(\frac{1-\left(v_{2}+v_{p}\right)^{q+1}}{v_{1}}\right)\right\} \\
& +\sum_{r=1}^{n_{r}} p_{23, r}\left(1-\left(\frac{r}{r+1}\right) \frac{v_{p}}{1-v_{2}}\right)\left(\frac{v_{p}}{1-v_{2}}\right)^{r} \\
& \left.\left.\times\left(\frac{1-\left(v_{1}+v_{p}\right)^{r+1}}{v_{2}}\right)\right\}\right] \frac{(i=1, \cdots, m)}{r=1} \chi_{23}^{0} v_{2}\left\{1-v_{p}\left(1+\sum_{r . r}^{n_{r}} v_{p}^{r}\right)\right.
\end{aligned}
$$

$\chi_{23}$, respectively, through the equations (see, Appendix B).

$$
\begin{aligned}
& g_{12}=\chi_{12} \\
& g_{13}=\frac{1}{v_{1}} \int_{1-v_{1}}^{1} \chi_{13} \mathrm{~d}\left(1-v_{1}\right) \\
& g_{23}=\frac{1}{v_{2}} \int_{1-v_{2}}^{1} \chi_{23} \mathrm{~d}\left(1-v_{2}\right)
\end{aligned}
$$

Equation 20 and 21 are readily written in the differential forms.

$$
\begin{aligned}
& \chi_{13}=g_{13}-v_{1} \frac{\partial g_{13}}{\partial\left(1-v_{1}\right)} \\
& \chi_{23}=g_{23}-v_{2} \frac{\partial g_{23}}{\partial\left(1-v_{2}\right)}
\end{aligned}
$$

Conditions of two-phase equilibrium of the quasi-ternary system at constant temperature and pressure are given by eq $24-26$,

$$
\begin{aligned}
\Delta \mu_{1(1)} & =\Delta \mu_{1(2)} \\
\Delta \mu_{2(1)} & =\Delta \mu_{2(1)} \\
\Delta \mu_{X_{i}(1)} & =\Delta \mu_{X_{i}(2)}
\end{aligned}
$$

where subscripts (1) and (2) denote the polymer-lean and -rich phases, respectively. Partition coefficient $\sigma$ is defined by:

$$
\sigma=\frac{1}{X_{i}} \ln \frac{v_{X_{i}(2)}}{v_{X_{i}(1)}}
$$

Combination of eq 15, 26, and 27 gives

For quasi-binary system, Koningsveld et $a l .{ }^{16,17}$ defined the thermodynamic interaction parameter $g$ in the expression of $\Delta G$. For quasi-ternary system, thermodynamic interaction parameters between solvent $1-2$, solvent 1-polymer, and solvent 2-polymer $g_{12}$, $g_{13}$, and $g_{23}$ can also be defined as,

$$
\begin{aligned}
& g_{12}=\Delta G_{12}^{E} /\left(\tilde{R} T L v_{1} v_{2}\right) \\
& g_{13}=\Delta G_{13}^{E} /\left(\tilde{R} T L v_{1} v_{p}\right) \\
& g_{23}=\Delta G_{23}^{E} /\left(\tilde{R} T L v_{2} v_{p}\right)
\end{aligned}
$$

$g_{12}, g_{13}$, and $g_{23}$ are related to $\chi_{12}, \chi_{13}$, and

$$
\begin{aligned}
\sigma= & \left(v_{p(1)}-v_{p(2)}\right)+\left(\frac{v_{p(2)}}{X_{n(2)}}-\frac{v_{p(1)}}{X_{n(1)}}\right) \\
& +\chi_{12}^{0}\left[v_{1(2)} v_{2(2)}-v_{1(1)} v_{2(1)}\right) \\
& -\sum_{s=1}^{n_{s}} p_{12, s} s\left\{v_{1(2)} v_{2(2)} v_{p(2)}^{s-1}\left(1-\frac{s+1}{s} v_{p(2)}\right)\right. \\
& \left.\left.-v_{1(1)} v_{2(1)} v_{p(1)}^{s-1}\left(1-\frac{s+1}{s} v_{p(1)}\right)\right\}\right] \\
& -\chi_{13}^{0}\left[\left(v_{1(2)}-v_{1(1)}\right)-\left(v_{1(2)} v_{p(2)}-v_{1(1)} v_{p(1)}\right)\right. \\
& -\sum_{q=1}^{n_{q}} p_{13, q}\left(v_{1(2)} v_{\cdot p(2)}^{q+1}-v_{1(1)} v_{p(1)}^{q+1}\right)
\end{aligned}
$$


Phase Equilibria of Quasi-Ternary Systems V

$$
\begin{aligned}
& +\sum_{q=1}^{n_{q}} \frac{p_{13, q}}{q+1}\left\{v_{1(2)}\left(q+1-\frac{q v_{p(2)}}{1-v_{1(2)}}\right)\right. \\
& \times\left(\frac{v_{p(2)}}{1-v_{1(2)}}\right)^{q} \frac{1-\left(v_{2(2)}+v_{p(2)}\right)^{q+1}}{v_{1(2)}} \\
& -v_{1(1)}\left(q+1-\frac{q v_{p(1)}}{1-v_{1(1)}}\right)\left(\frac{v_{p(1)}}{1-v_{1(1)}}\right)^{q} \\
& \left.\left.\times \frac{1-\left(v_{2(1)}+v_{p(1)}\right)^{q+1}}{v_{1(1)}}\right\}\right] \\
& -\chi_{23}^{0}\left[\left(v_{2(2)}-v_{2(1)}\right)-\left(v_{2(2)} v_{p(2)}-v_{2(1)} v_{p(1)}\right)\right. \\
& -\sum_{r=1}^{n_{r}} p_{23, r}\left(v_{2(2)} v_{p(2)}^{r+1}-v_{2(1)} v_{p(1)}^{r+1}\right) \\
& +\sum_{r=1}^{n_{r}} \frac{p_{23, r}}{r+1}\left\{v_{2(2)}\left(r+1-\frac{r v_{p(2)}}{1-v_{2(2)}}\right)\right. \\
& \times\left(\frac{v_{p(2)}}{1-v_{2(2)}}\right)^{r} \frac{1-\left(v_{1(2)}+v_{p(2)}\right)^{r+1}}{v_{2(2)}} \\
& -v_{2(1)}\left(r+1-\frac{r v_{p(1)}}{1-v_{2(1)}}\right)\left(\frac{v_{p(1)}}{1-v_{2(1)}}\right)^{r} \\
& \left.\left.\times \frac{1-\left(v_{1(1)}+v_{p(1)}\right)^{r+1}}{v_{2(1)}}\right\}\right]
\end{aligned}
$$

Substitution of eq 13 into 24 yields eq 29 (the left-hand side of eq 29 is put with $A$ ),

$$
\begin{aligned}
A \equiv & \ln \frac{v_{1(2)}}{v_{1(1)}}+\left(v_{p(2)}-v_{p(1)}\right)-\left(\frac{v_{p(2)}}{X_{n(2)}}-\frac{v_{p(1)}}{X_{n(1)}}\right) \\
& +\chi_{12}^{0}\left[\left(v_{2(2)}-v_{2(1)}\right)-\left(v_{1(2)} v_{2(2)}-v_{1(1)} v_{2(1)}\right)\right. \\
& +\sum_{s=1}^{n_{s}} p_{12, s}\left\{\left(v_{2(2)} v_{p(2)}^{s}-v_{2(1)} v_{p(1)}^{s}\right)\right. \\
& \left.\left.-(s+1)\left(v_{1(2)} v_{2(2)} v_{p(2)}^{s}-v_{1(1)} v_{2(1)} v_{p(1)}^{s}\right)\right\}\right] \\
& +\chi_{13}^{0}\left[\left(v_{p(2)}-v_{p(1)}\right)-\left(v_{1(2)} v_{p(2)}-v_{1(1)} v_{p(1)}\right)\right. \\
& +\sum_{q=1}^{n_{q}} p_{13, q}\left\{v_{p(2)}^{q+1}\left(1-v_{1(2)}\right)\right. \\
& \left.\left.-v_{p(1)}^{q+1}\left(1-v_{1(1)}\right)\right\}\right]
\end{aligned}
$$

$$
\begin{aligned}
& -\chi_{23}^{0}\left[\left(v_{2(2)} v_{p(2)}-v_{2(1)} v_{p(1)}\right)\right. \\
& +\sum_{r=1}^{n_{r}} p_{23, r}\left(v_{2(2)} v_{p(2)}^{r+1}-v_{2(1)} v_{p(1)}^{r+1}\right) \\
& +\sum_{r=1}^{n_{r}} p_{23, r}\left(\frac{r}{r+1}\right)\left\{v_{2(2)}\left(\frac{v_{p(2)}}{1-v_{p(2)}}\right)^{r+1}\right. \\
& \times \frac{1-\left(v_{1(2)}+v_{p(2)}\right)^{r+1}}{v_{2(2)}}-v_{2(1)}\left(\frac{v_{p(1)}}{1-v_{p(1)}}\right)^{r+1} \\
& \left.\left.\times \frac{1-\left(v_{1(1)}+v_{p(1)}\right)^{r+1}}{v_{2(1)}}\right\}\right]=0
\end{aligned}
$$

Substitution of eq 14 into 25 gives eq 30 (the left-hand side of eq 30 is put with $B$ ):

$$
\begin{aligned}
& B \equiv \ln \frac{v_{2(2)}}{v_{2(1)}}+\left(v_{p(2)}-v_{p(1)}\right)-\left(\frac{v_{p(2)}}{X_{n(2)}}-\frac{v_{p(1)}}{X_{n(1)}}\right) \\
&+\chi_{12}^{0}\left[\left(v_{1(2)}-v_{1(1)}\right)-\left(v_{1(2)} v_{2(2)}-v_{1(1)} v_{2(1)}\right)\right. \\
&+\sum_{s=1}^{n_{s}} p_{12, s}\left\{\left(v_{1(2)} v_{p(2)}^{s}-v_{1(1)} v_{p(1)}^{s}\right)\right. \\
&\left.\left.-(s+1)\left(v_{1(2)} v_{2(2)} v_{p(2)}^{s}-v_{1(1)} v_{2(1)} v_{p(1)}^{s}\right)\right\}\right] \\
&+\chi_{23}^{0}\left[\left(v_{p(2)}-v_{p(1)}\right)-\left(v_{2(2)} v_{p(2)}-v_{2(1)} v_{p(1)}\right)\right. \\
&\left.+\sum_{r=1}^{n_{r}} p_{23, r}\left\{v_{p(2)}^{r+1}\left(1-v_{2(2)}\right)-v_{p(1)}^{r+1}\left(1-v_{2(1)}\right)\right\}\right] \\
&-\chi_{13}^{0}\left[\left(v_{1(2)} v_{p(2)}-v_{1(1)} v_{p(1)}\right)\right. \\
&+\sum_{q=1}^{n_{q}} p_{13, q}\left(v_{1(2)} v_{p(2)}^{q+1}-v_{1(1)} v_{p(1)}^{q+1}\right) \\
&+\sum_{q=1}^{n_{q}} p_{13, q}\left(\frac{q}{q+1}\right)\left\{v_{1(2)}\left(\frac{v_{p(2)}}{1-v_{p(2)}}\right)^{q+1}\right. \\
& \times \frac{1-\left(v_{2(2)}+v_{p(2)}\right)^{q+1}}{v_{1(2)}}-v_{1(1)}\left(\frac{v_{p(1)}}{1-v_{p(1)}}\right)^{q+1} \\
& \frac{1-\left(v_{2(1)}+v_{p(1)}\right)^{q+1}}{\left.\left.v_{1(1)}\right\}\right]=0}(30) \\
& \frac{q}{q}(a) \chi_{0} \\
&
\end{aligned}
$$

If both (a) $\chi_{12}^{0}, \chi_{13}^{0}$, and $\chi_{23}^{0}$ and (b) $p_{12, s}, p_{13, q}$, and $p_{23, r}\left(s=1, \cdots, n_{s} ; q=1, \cdots, n_{q} ; r=1, \cdots, n_{r}\right)$ are given in advance, $\sigma, A$, and $B$ are functions 
having six variables, $X_{n(1)}, X_{n(2)}, v_{2(1)}, v_{2(2)}, v_{p(1)}$, and $v_{p(2)}$.

Dissolve original polymer into solvent (ordinally, solvent 1) and add non-solvent (ordinally, solvent 2), two-phase separation finally occurs. If volumes of solvent 1,2 , and polymer are $V_{1}^{0}, V_{2}$, and $V_{3}^{0}$, respectively, the volume of starting solution $V_{0}$ is given by $V_{1}^{0}+V_{3}^{0}$ and the starting concentration $v_{p}^{s}$ is given by

$$
v_{p}^{s}=\frac{V_{3}^{0}}{V_{0}}=\frac{V_{3}^{0}}{V_{1}^{0}+V_{3}^{0}}
$$

Under the two phase equilibrium, the total volume of the system is $V_{1}^{0}+V_{2}+V_{3}^{0}(=V)$ and the initial concentration $v_{p}^{0}$ is expressed by

$$
v_{p}^{0}=\frac{V_{3}^{0}}{V}=\frac{V_{3}^{0}}{V_{1}^{0}+V_{2}+V_{3}^{0}}
$$

Normalized molecular weight distribution (MWD) of original polymer $g_{0}\left(X_{i}\right)$ is a summation of - the relative amounts of $X_{i}$-mer separated into polymer-lean and -rich phase, $g_{(1)}\left(X_{i}\right)$ and $g_{(2)}\left(X_{i}\right)$ :

$g_{0}\left(X_{i}\right)=g_{(1)}\left(X_{i}\right)+g_{(2)}\left(X_{i}\right) \quad(i=1, \cdots, m)$

The weight fraction $\rho_{s}$ of the polymer in the polymer-lean phase to the total polymer and the fraction $\rho_{p}\left(=1-\rho_{s}\right)$ of the polymer in the polymer-rich phase are given by eq $34 \mathrm{a}$ and $34 \mathrm{~b}$.

$$
\begin{aligned}
& \rho_{s}=\sum_{i=1}^{m} g_{(1)}\left(X_{i}\right) \\
& \rho_{p}=\sum_{i=1}^{m} g_{(2)}\left(X_{i}\right)
\end{aligned}
$$

Using $\sigma$, phase volume ratio $R\left(=V_{(1)} / V_{(2)}\right.$; $V_{(1)}$ and $V_{(2)}$, volume of the polymer-lean and rich phases) and $g_{0}\left(X_{i}\right), g_{(1)}\left(X_{i}\right)$, and $g_{(2)}\left(X_{i}\right)$ are expressed as (see, Appendix $C$ ):

$$
\begin{aligned}
& g_{(1)}\left(X_{i}\right)=\frac{R}{R+\exp \left(\sigma X_{i}\right)} g_{0}\left(X_{i}\right) \\
& g_{(2)}\left(X_{i}\right)=\frac{\exp \left(\sigma X_{i}\right)}{R+\exp \left(\sigma X_{i}\right)} g_{0}\left(X_{i}\right)
\end{aligned}
$$

$v_{p(1)}, v_{p(2)}$, and $X_{n(1)}$ are finally expressed by eq $36 \mathrm{a}, \mathrm{b}$, and 37 (see, Appendix C).

$$
\begin{aligned}
v_{p(1)}= & v_{p}^{s} \frac{R+1-R v_{2(1)}-v_{2(2)}}{R} \rho_{s} \\
v_{p(2)}= & v_{p}^{s}\left(R+1-R v_{2(1)}-v_{2(2)}\right) \rho_{p} \\
& \frac{1}{X_{n(1)}}=\frac{1}{\rho_{s}}\left(\frac{1}{X_{n}^{0}}-\frac{1}{X_{n(2)}}\right)
\end{aligned}
$$

Here, $X_{n}^{0}$ is the number-average $X_{i}$ of original polymer. If $\rho_{p}$ is set as initial condition $\rho_{p}^{g}$, $\sigma, A$, and $B$ become the functions of four variables $v_{2(1)}, v_{2(2)}, R^{a}$, and $X_{n(2)}^{a}$.

$$
\begin{aligned}
& \sigma=\sigma\left(v_{2(1)}, v_{2(2)}, R^{a}, X_{n(2)}^{a}\right) \\
& A=A\left(v_{2(1)}, v_{2(2)}, R^{a}, X_{n(2)}^{a}\right)=0 \\
& B=B\left(v_{2(1)}, v_{2(2)}, R^{a}, X_{n(2)}^{a}\right)=0
\end{aligned}
$$

where $R^{a}$ and $X_{n(2)}^{a}$ are the assumed value of $R$ and $X_{n(2)} . X_{n(2)}$ in eq $38-40$ is given by

$$
X_{n(2)}=\sum_{i=1}^{m} g_{(2)}\left(X_{i}\right) /\left(\sum_{i=1}^{m} g_{(2)}\left(X_{i}\right) / X_{i}\right)
$$

Combining eq $34 \mathrm{~b}, 35 \mathrm{~b}$, and $41, \rho_{p}$ and $X_{n(2)}$ are finally the functions of $\sigma$ and $R^{a}$. We define $C$ and $D$ by eq 42 and 43 , respectively.

$$
\begin{aligned}
& C \equiv \rho_{p}\left(\sigma\left(v_{2(1)}, v_{2(2)}, R^{a}, X_{n(2)}^{a}\right), R^{a}\right)-\rho_{p}^{g} \\
& D \equiv X_{n(2)}\left(\sigma\left(v_{2(1)}, v_{2(2)}, R^{a}, X_{n(2)}^{a}\right), R^{a}\right)-X_{n(2)}^{a}
\end{aligned}
$$

By solving non-linear simultaneous equations $39,40,42$, and $43, v_{2(1)}, v_{2(2)}, R$, and $X_{n(2)}$ are determined. Substituting these four values into eq 38, we can calculate $\sigma$ and other phase separation characteristics (see, Appendix C).

\section{COMPUTER SIMULATION}

Computer experiments were carried out according to the procedure, established in the previous papers. ${ }^{9-11}$

1. As prerequisites, (a) $\chi_{12}^{0}, \chi_{13}^{0}$, and $\chi_{23}^{0}$, (b) $p_{12, s}, p_{13, q}$, and $p_{23, r}\left(s=1, \cdots, n_{s} ; q=1, \cdots\right.$, 
$\left.n_{q} ; r=1, \cdots, n_{r}\right)$, (c) $V_{1}^{0}, V_{3}^{0}, g_{0}\left(X_{i}\right)(i=1, \cdots$, $m)$, and $\rho_{p}\left(=1-\rho_{s}\right)$ are given.

2. At first, $X_{n(2)}^{a}$ and three values of $R^{a}$ (low, middle, and high value of $R^{a}, R_{L}, R_{M}$, and $R_{H}$, respectively) are assumed. True $\mathrm{R}$ should be between $R_{L}$ and $R_{M}$ or $R_{M}$ and $R_{H}$.

3 . For the assumed $X_{n(2)}$ and $R^{a}$, simultaneous equations 44 and 45 ,

$$
A=A\left(v_{2(1)}, v_{2(2)}\right)=0
$$

$$
B=B\left(v_{2(1)}, v_{2(2)}\right)=0
$$

are solved by using two variable Newton's method.

4. Substituting three sets of $\left(v_{2(1)}, v_{2(2)}\right)$, which correspond to $R_{L}, R_{M}$, and $R_{H}$, into eq 42 , we get $C_{L}, C_{M}$, and $C_{H}$. Reset $\left(R_{L}, R_{H}\right)$ by $\left(R_{L}, R_{M}\right)$ for $C_{L} \cdot C_{M}<0$ and by $\left(R_{M}, R_{H}\right)$ for $C_{M} \cdot C_{H}<0 . \sigma_{L}, \sigma_{M}$, and $\sigma_{H}$ are also obtained.

5. Using interhalving method (repetition

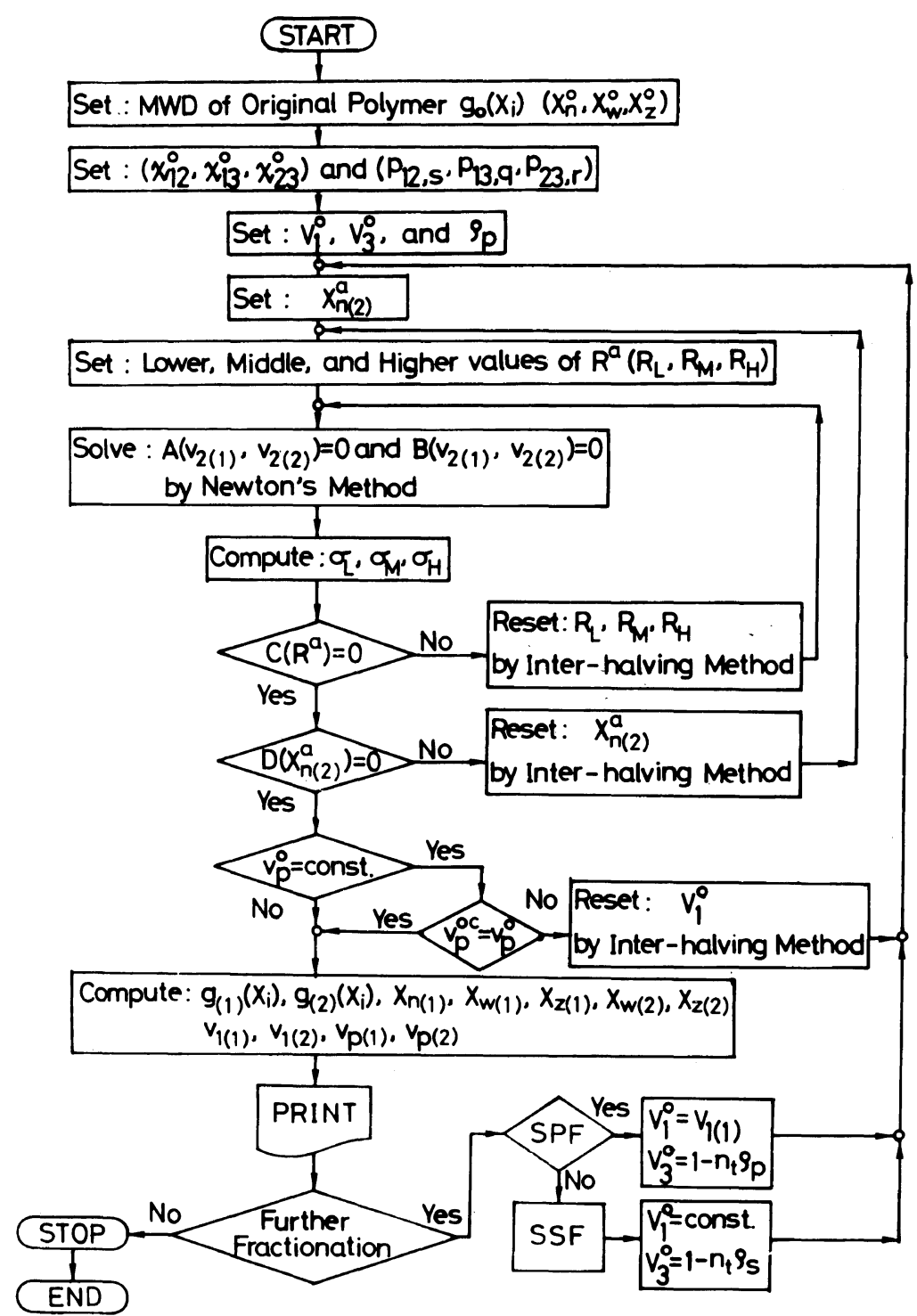

Figure 1. Flow chart of the simulation. 
of step 3-4),

$$
C=C\left(R^{a}\right)=0
$$

can be attained. Thus, $R$ and $\sigma$ (accordingly, $g_{(1)}\left(X_{i}\right)$ and $\left.g_{(2)}\left(X_{i}\right)\right)$ are determined for the assumed value of $X_{n(2)}$.

6 . When $\mathrm{D}$ (given by eq 43 ) $\neq 0$ for calculated $\sigma$ and $R$, replace $X_{n(2)}^{a}$ by $\left(X_{n(2)}+X_{n(2)}^{a}\right) / 2$.

7. Repeating step 3-6 (interhalving method),

$$
D=D\left(X_{n(2)}^{a}\right)=0
$$

can be solved for $X_{n(2)}^{a}$ and therefore an equilibrium state is finally determined.

8. When a constant initial polymer volume fraction $\left(v_{p}^{0}=\right.$ constant, $\left.v_{p}^{0 g}\right)$ is needed, we solve the following equation

$$
v_{p}^{0}\left(v_{2(1)}, v_{2(2)}, R^{a}, V_{1}^{0}\right)-v_{p}^{0 g}=0
$$

by replacing $V_{1}^{0}$ (repetition of step $1-7$ ).

9. Compute other phase separation characteristics (a) $V_{2}, V, V_{(1)}$, and $V_{(2)}$, (b) $v_{1(1)}$, $v_{1(2)}, v_{p(1)}$, and $v_{p(2)}$, and (c) $X_{n(1)}, X_{w(1)}, X_{w(2)}$ and so on.

This simulation procedure is superior to others ${ }^{1-7,13}$ with respect to following point.

(i) MWD of polymer in both phases can directly be obtained, in addition to the volume fractions of solvents and polymer.

(ii) $\rho_{p}$ can be determined rigorously. For example, Altena and Smolders ${ }^{13}$ did not write clearly $\rho_{p}$ in their computer experiments on the phase separation of mono-disperse polymer/binary solvent mixture.

(iii) Calculations under constant $v_{p}^{0}$ are feasible. Constant $v_{p}^{0}$ is important to compare quasi-ternary system with quasi-binary system. Figure 1 shows the main flow chart of simulation.

\section{COMPUTER EXPERIMENT}

Original polymer was assumed to have the Schulz-Zimm (SZ) type MWD,

$$
g_{0}\left(X_{i}\right)=\frac{y^{h+1}}{\Gamma(h+1)} X_{i}^{h} \exp \left(-y X_{i}\right)
$$

where

$$
y=h / X_{n}^{0}
$$

and

$$
h=1 /\left(X_{w}^{0} / X_{n}^{0}-1\right)
$$

with $X_{w}^{0}=300$ and $X_{w}^{0} / X_{n}^{0}=2 . \Gamma(h+1)$ is the gamma function. We have already ascertained that the conclusion obtained for the SZ type polymer with $X_{w}^{0}=300$ has very general character applicable for common polymer solution. ${ }^{11}$ The calculations were made under following conditions: (a) $\chi_{12}^{0}=0.5$, $\chi_{13}^{0}=0.2$, and $\chi_{23}^{0}=1.0$, (b) $p_{12,1}=-1.0 \sim 2.0$, $p_{13,1}=-0.6 \sim 0.6$, and $p_{23,1}=-0.15 \sim 0.15$ $\left(p_{12, s}=p_{13, q}=p_{23, r}=0\right.$ for $\left.\mathrm{s}, \mathrm{q}, \mathrm{r} \geqq 2\right)$, (c) $\rho_{p}=$ $1 / 100 \sim 99 / 100, V_{3}^{0}=1$, and $v_{p}^{0}=0.005$. For comparison, calculations of quasi-binary system, under the conditions of $p_{1}=-0.6$, 0 , and $0.6\left(p_{j}=0, j \geqq 2\right), V_{3}^{0}=1$, and $v_{p}^{0}=0.005$, were also carried out. When coexisting curves were calculated, we put $v_{p}^{s}=0.01$ in place of $v_{p}^{0}=0.005$. Equations $46-48$ were considered to be solved, if $\left|C\left(R^{a}\right)\right|<E_{1},\left|D\left(X_{n(2)}^{a}\right)\right|$ $<E_{2}$, and $\left|v_{p}^{0}-v_{p}^{0 g}\right|<E_{3}$ were satisfied. $E_{1}$, $E_{2}$, and $E_{3}$ are allowance errors. Here, $E_{1}=$ $0.001, E_{2}=0.01$, and $E_{3}=0.001$.

\section{RESULTS AND DISCUSSION}

Figure 2 shows the effects of $p_{12,1}, p_{13,1}$, and $p_{23,1}$ on the normalized $X$ distribution of the polymer partitioned in the polymer-rich phase $g_{(2)}\left(X_{i}\right)$ at $\rho_{p}=1 / 15 . p_{12,1}$ was found to have small but still significant effect on $g_{(2)}\left(X_{i}\right)$ and the breadth in $g_{(2)}(X)$ attains minimum at $p_{12,1}=-0.2$ when $p_{13,1}=p_{23,1}=0$. The polydispersity of the polymer in a polymer-rich phase becomes lower with an increase in $p_{13,1}$ or $p_{23,1}$, at least in the range $p_{13,1} \leqq 0.6$ and $p_{23,1} \leqq 0.07$. Particularly, a small change in 


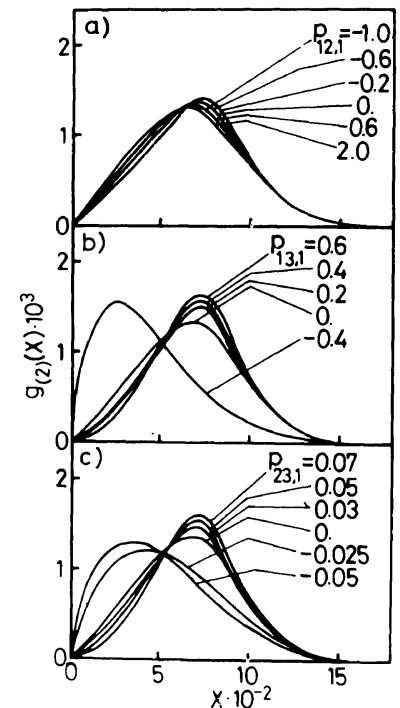

Figure 2. Effects of the 1st order concentration dependent parameters $p_{12,1}, p_{13.1}$, and $p_{23,1}$ on the normalized molecular weight distribution of the polymer partitioned in polymer rich phase $g_{(2)}$. Original polymer, Schulz-Zimm type distribution $\left(X_{w}^{0}=300, X_{w}^{0} / X_{n}^{0}=2\right)$; $\chi_{12}^{0}=0.5, \chi_{13}^{0}=0.2$, and $\chi_{23}^{0}=1.0, \rho_{p}=1 / 15$ and $v_{p}^{0}=$ 0.005. a) $p_{13,1}=p_{23,1}=0$, b) $p_{12,1}=p_{23,1}=0$, c) $p_{12,1}=$ $p_{13,1}=0$.

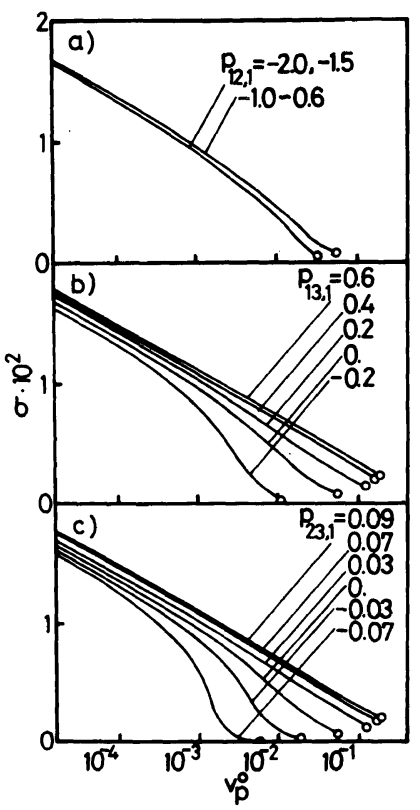

Figure 3. Effects of the 1st order concentration dependent parameters $p_{12,1}, p_{13,1}$ and $p_{23,1}$ on the relations between partition coefficient $\sigma$ and $v_{p}^{0}$. Original polymer, Schulz-Zimm type distribution $\left(X_{w}^{0}=300, X_{w}^{0} / X_{n}^{0}=2\right)$; $\chi_{12}^{0}=0.5, \chi_{13}^{0}=0.2$, and $\chi_{23}^{0}=1.0, \rho_{p}=1 / 15$. a) $p_{13,1}=$ $p_{23,1}=0$, b) $\dot{p}_{12,1}=\dot{p}_{23,1}=0$, c) $p_{12,1}=p_{13,1}=0$. Unfilled circle denotes $v_{p, c}^{0}$.

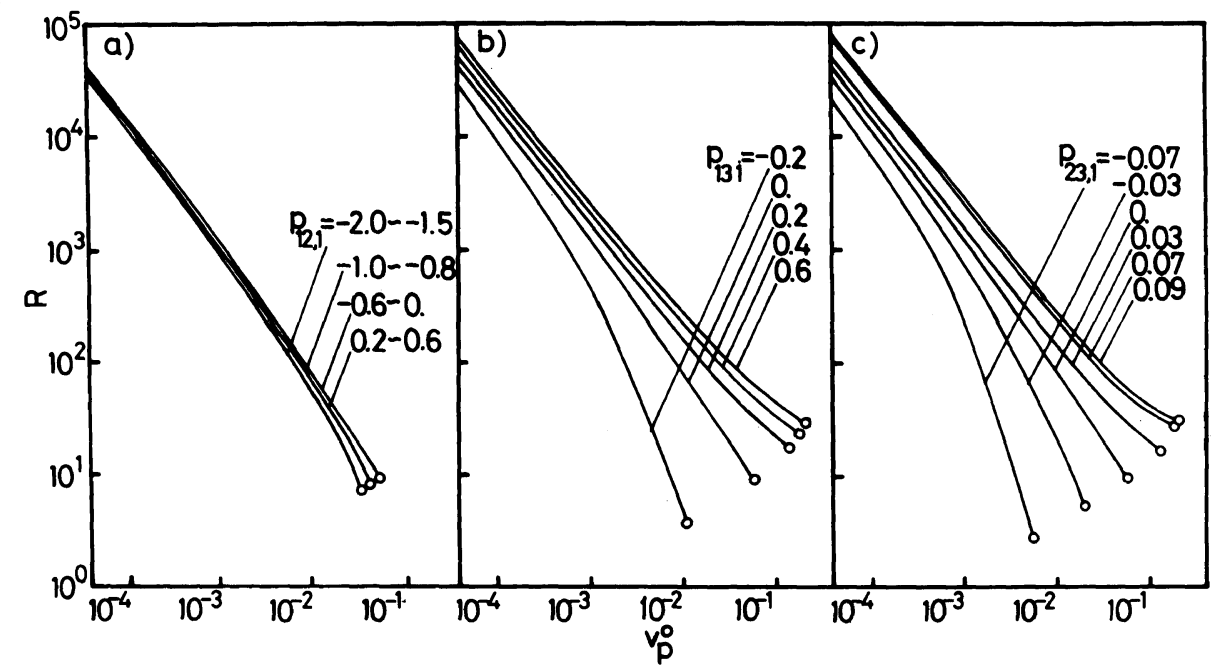

Figure 4. Effects of $p_{12,1}, p_{13,1}$, and $p_{23,1}$ on the relations between the two phase volume ratio $R$ and $v_{p}^{0}$. Original polymer, Schulz-Zimm type distribution $\left(X_{w}^{0}=300, X_{w}^{0} / X_{n}^{0}=2\right) ; \chi_{12}^{0}=0.5, \chi_{13}^{0}=0.2$, and $\chi_{23}^{0}=1.0$, $\rho_{p}=1 / 15$. a) $p_{13,1}=p_{23,1}=0$, b) $p_{12,1}=p_{23,1}=0$, c) $p_{12,1}=p_{13,1}=0$. Unfilled circle denotes $v_{p, c}^{0}$. 
$p_{23,1}$ brings about a large change in $g_{(2)}(X)$. The effect of the concentration dependence of $\chi$-parameters on $g_{(2)}(X)$ decreases in the following order: $p_{23,1}>p_{13,1}>p_{12,1}$.

In Figures 3 and 4 , the effects of $p_{12,1}, p_{13,1}$, and $p_{23,1}$ on the $\sigma$ (or $R$ ) vs. $v_{p}^{0}$ relations at $\rho_{p}=1 / 15$ are shown. Unfilled circle indicates the point at which both $\sigma$ and $R$ reach a minimum. Above $v_{p}^{0}$ at minimum, twophase separation under given conditions becomes impossible and this $v_{p}^{0}$ was defined as "critical point" by Kamide et al. ${ }^{18}$ for quasibinary system and referred to as $v_{p, c}^{0}$. Unfilled circle approaches to critical solution point (CSP) in the limit of $\rho_{p} \rightarrow 0$. Volume fractions

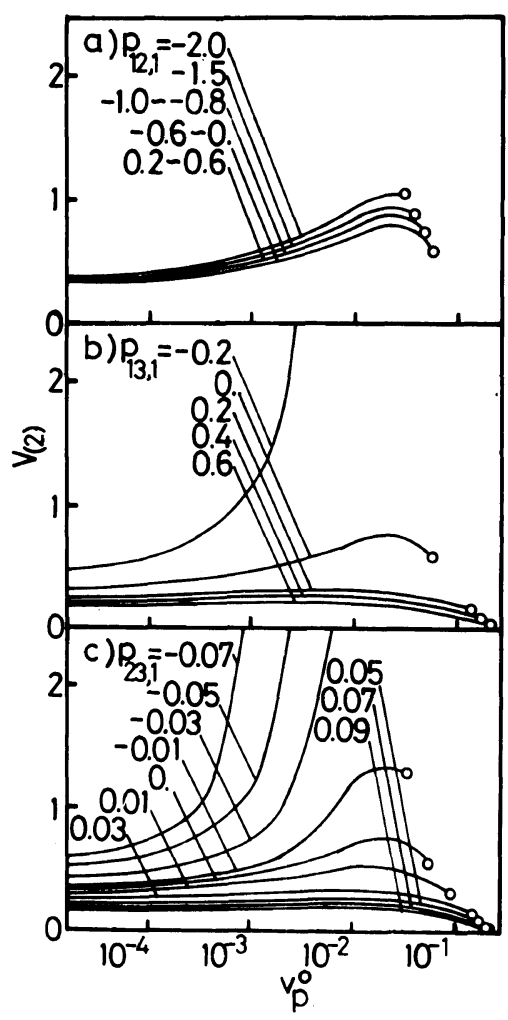

Figure 5. Effects of $p_{12,1}, p_{13,1}$, and $p_{23,1}$ on the relations between the volume of the polymer rich phase $V_{(2)}$ and $v_{p}^{0}$. Original polymer, Schulz-Zimm type distribution $\left(X_{w}^{0}=300, X_{w}^{0} / X_{n}^{0}=2\right) ; \chi_{12}^{0}=0.5, \chi_{13}^{0}=0.2$, and $\chi_{23}^{0}=1.0, \rho_{p}=1 / 15$. a) $p_{13,1}=p_{23,1}=0$, b) $p_{12,1}=p_{23,1}=0$, c) $p_{12,1}=p_{13,1}=0$. Unfilled circle denotes $v_{p, c}^{c}$. of solvents 1,2 , and polymer at $\operatorname{CSP}\left(v_{1}^{c}, v_{2}^{c}\right.$, and $v_{p}^{c}$, respectively) can be calculated from the spinodal and neutral-equilibrium conditions according to the method proposed by Kamide and Matsuda. ${ }^{19}\left(v_{1}^{c}, v_{2}^{c}, v_{p}^{c}\right)$ is $(0.4880$, $0.4609,0.0511)$ under the conditions of $\left(\chi_{12}^{0}\right.$, $\left.\chi_{13}^{0}, \chi_{23}^{0}\right)=(0.5,0.2,1.0)$ with $p_{12,1}=p_{13,1}=$ $p_{23,1}=0$. When $p_{12,1}=p_{13,1}=p_{23,1}=0, \quad v_{p, c}^{0}$ at $\rho_{p}=1 / 15$ is $c a .0 .05$ and is close to $v_{p}^{c}$. Both $\sigma$ and $R$ decrease with an increase in $v_{p}^{0}$. Generally, $\sigma$ and $R$, at given $\rho_{p}$, increase with increasing $p_{13,1}$, and $p_{23,1}$. The effects of $p_{12,1}$ on $\sigma$ (or $R$ ) vs. $v_{p}^{0}$ curve are small.

Relations between $V_{(2)}$ and $v_{p}^{0}$ under the conditions of $\rho_{p}=1 / 15$ are shown in Figure 5. In this figure, unfilled circle, is $v_{p, c}^{0}$. As $v_{p}^{0}$ increases, $V_{(2)}$ increases first and shows the maximum. $v_{p}^{0}$ at this maximum of $V_{(2)}$ vs. $v_{p}^{0}$ curve, corresponds to $v_{p}^{0}$, which gives the maximum of $v_{1(2)}$ or $v_{2(2)} v s$. $v_{p}^{0}$ curve and also corresponds to the minimum of $v_{p(2)} v s . v_{p}^{0}$ curve (for example, see Figure 13). With a decrease in $p_{13,1}$ and $p_{23,1} V_{(2)}$ increases suddenly. The effects of $p_{12,1}$ on $V_{(2)}$ is relatively small. And $V_{(2)}$ shows a minimum at a specific $p_{12,1}$, between $p_{12,1}=-0.6$ and 0 . On the other hand, $V$ and $V_{(1)}$ decrease almost linearly with an increase in $v_{p}^{0}$. $V_{(1)} / V_{(2)}$ (namely $R$ ) vs. $v_{p}^{0}$ curve is little influenced by the maximum of $V_{(2)}$ vs. $v_{p}^{0}$ curve because of a rapid change in $V_{(1)}$ (see, Figure 4).

Figure 6 shows the effects of $p_{12,1}, p_{13,1}$, and $p_{23,1}$ on the relations between $V_{1}$ (or $V_{2}$ ) and $\rho_{p}$ relations, when $v_{p}$ is constant (in this case, $v_{p}^{0}=0.005$ ). At constant $v_{p}^{0}$ (namely, constant $V$ ), we can compare $p_{12,1}, p_{13,1}$, and $p_{23,1}$ with $p_{1}$, which is the concentration dependent parameter of $\chi$ for quasi-binary solutions (see eq 4). Between $V_{1}$ vs. $\rho_{p}$ curve and $V_{2} v s . \rho_{p}$ curve, there is a mirror symmetry with respect to the broken line of $V_{1}=V_{2}=$ $\left(V-V_{3}^{0}\right) / 2$ (in this case $V=200$ and $V_{3}^{0}=1$ ). As $p_{13,1}$ and $p_{23,1}$ increase $V_{1}$ decreases and $V_{2}$ increases. In other words, solvent power of solvent 1 grows stronger with an increase in $p_{13,1}$, and precipitating power of solvent 2 
Phase Equilibria of Quasi-Ternary Systems V.

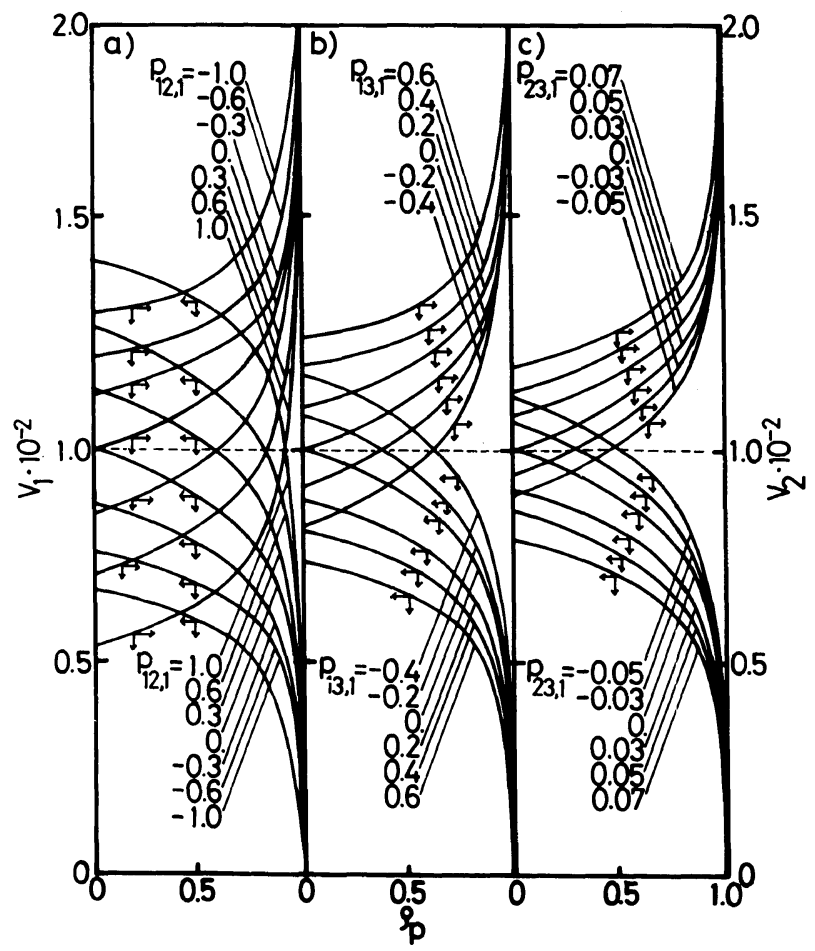

Figure 6. Effects of $p_{12,1}, p_{13,1}$, and $p_{23,1}$ on the relations of the volume of solvent 1 (or 2), $V_{1}^{0}$ (or $V_{2}$ ) and $\rho_{p}$. Original polymer, Schulz-Zimm type distribution $\left(X_{w}^{0}=300, X_{w}^{0} / X_{n}^{0}=2\right) ; \chi_{12}^{0}=0.5, \chi_{13}^{0}=0.2$, and $\chi_{23}^{0}=$ 1.0, $v_{p}^{0}=0.005$. a) $p_{13,1}=p_{23,1}=0$, b) $p_{12,1}=p_{23,1}=0$, c) $p_{12,1}=p_{13,1}=0$. Broken line denotes the line of $V_{1}=$ $V_{2}\left(=\left(V-V_{3}^{0}\right) / 2\right)$.

gets weaker with an increase in $p_{23,1}$. As $p_{12,1}$, decreases, solvent power of binary solvent mixture becomes stronger. The figure shows that $V_{1}$ reduces to zero at $\rho_{p}=1.0$.

The effects of $p_{12,1}, p_{13,1}$, and $p_{23,1}$ on the relations between $\sigma$ (or $R$ ) and $\rho_{p}$ are shown in Figure 7. In the figure, results of quasi-binary system with $p_{1}=-0.6,0$, and $0.6 \quad\left(p_{j}=0\right.$, $J \geqq 2$ ) are also shown by the broken line. ${ }^{20-27}$ In the case of $p_{12,1}, p_{13,1}$, and $p_{23,1}=0, \sigma$ and $R$ for quasi-ternary system are smaller than those for quasi-binary system with $p_{1}=0$ for any combination of $\chi_{12}^{0}, \chi_{13}^{0}$, and $\chi_{23}^{0} .{ }^{9}$ When $p_{13,1} \geqq 0.2$ (with $p_{12,1}=p_{23,1}=0$ ) or $p_{23,1} \geqq 0.03$ (with $p_{12,1}=p_{23,1}=0$ ), $\sigma$ (or $R$ ) for the quasi-ternary system exceeds $\sigma$ (or $R$ ) for the quasi-binary system with $p_{1}=0$. Especially, $\sigma$ and $R$ for the quasi-ternary system with $p_{23,1}=0.09\left(p_{12,1}=p_{13,1}=0\right)$ are larger than $\sigma$ and $R$ for the quasi-binary system with $p_{1}=0.6$. In consequence, under specific conditions, a better fractionation efficiency is expected to be obtained for the quasi-ternary solution than for the quasi-binary solutions. This prediction however has not been experimentally confirmed and clearly more detailed experimental examination is called for. The effect of $p_{12,1}$ on $\sigma$ (or $R$ ) is not so large.

We denote $\sigma, R$, and the volume fractions of each phase at $\rho_{p}=1$ with an asterisk as $\sigma^{*}, R^{*}$, $\left(v_{1(1)}^{*}, v_{2(1)}^{*}, v_{p(1)}^{*}\right)$ and $\left(v_{1(1)}^{*}, v_{2(2)}^{*}, v_{p(1)}^{*}\right) \cdot v_{p(2)}^{*}$ can be calculated by the following equation. 


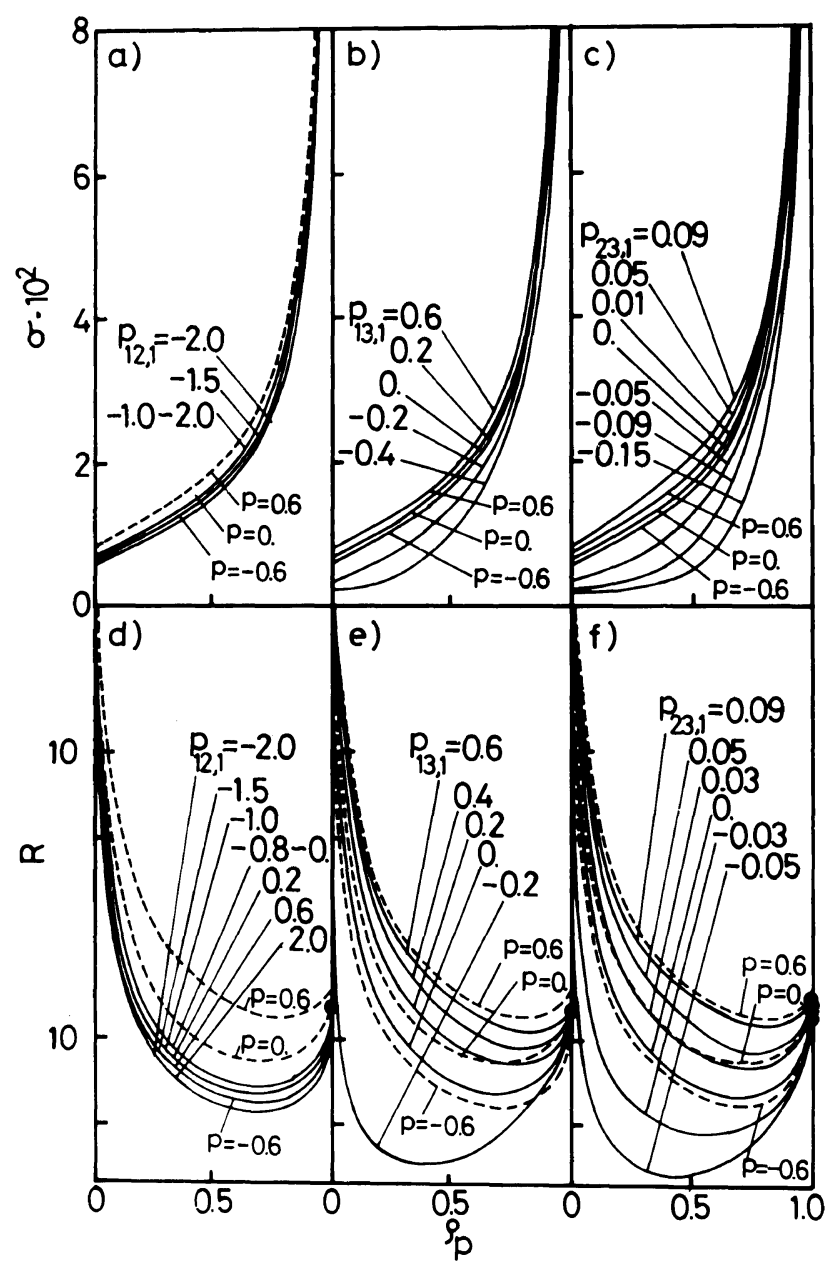

Figure 7. Effects of $p_{12,1}, p_{13,1}$, and $p_{23,1}$ on the relations between partition coefficient $\sigma$ or the phase volume ratio $R$ and $\rho_{p}$. Original polymer, Schulz-Zimm type distribution $\left(X_{w}^{0}=300, X_{w}^{0} / X_{n}^{0}=2\right) ; \chi_{12}^{0}=0.5$, $\chi_{13}^{0}=0.2$, and $\chi_{23}^{0}=1.0, v_{p}^{0}=0.005$. a) and d) $p_{13,1}=p_{23,1}=0$, b) and e) $p_{12,1}=p_{23,1}=0$, c) and f) $p_{12,1}=$ $p_{13,1}=0$. The broken lines are the results for quasi-binary system. $p$ is denoted on curve. Filled circle denotes $R$ at $\rho_{p}=1.0$.

$$
\begin{aligned}
\ln \left(1-v_{p(2)}^{*}\right)+v_{p(2)}^{*}-\frac{v_{p(2)}^{*}}{X_{n}^{0}} & \\
& +\chi_{23}^{0}\left[v_{p(2)}^{* 2}+\sum_{r=1}^{n_{r}} p_{23, r} v_{p(2)}^{* r+2}\right]=0
\end{aligned}
$$

and $R^{*}$ is expressed by

$$
R^{*}=\frac{v_{p(2)}^{*}}{v_{p}^{0}}-1
$$

As is known from eq 52 and $53, v_{p(2)}^{*}$ and
$R^{*}$ are independent of $\chi_{12}^{0}, \chi_{13}^{0}, p_{12, s}$, and $p_{13, q}$ $\left(s=1, \cdots, n_{s} ; q=1, \cdots, n_{q}\right)$ and determined by $\chi_{23}^{0}$ and $p_{23, r}\left(r=1, \cdots, n_{r}\right)$. The extrapolated values of $R$ for $\rho_{p} \rightarrow 1$ are given by filled circle in Figure $7 \mathrm{~d}$, e, and $\mathrm{f}$, and are consistent with $R^{*}$ calculated from eq 52. From the combination of $v_{x_{i}(1)}=0(i=1, \cdots, 0)$ for $\rho_{p}=1$ and the definition of $\sigma$ (eq 27), $\sigma$ for $\rho_{p}=1\left(\sigma^{*}\right)$ becomes infinite. Figure 8 shows the effect of $p_{23,1}$ on $R^{*}, v_{2(2)}^{*}$, and $v_{p(2)}^{*}$. With an increase in $p_{23,1}, R^{*}$ and $v_{p(2)}^{*}$ increase and $v_{2(2)}^{*}$ decreases. 
We can evaluate $\chi_{23}^{0}$ and $p_{23, r}$ from $R^{*}$ experimentally determined.

The effects of $p_{12,1}, p_{13,1}$, and $p_{23,1}$ on relations between $X_{w} / X_{n}$ and $v_{p}^{0}$ (for $\rho_{p}=1$ ) 15) are shown in Figure 9. With an increase in $p_{13,1}$ and $p_{23,1}, X_{w} / X_{n}$ decreases abruptly.

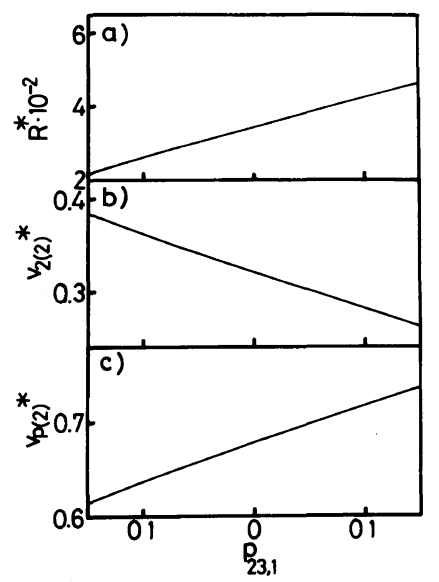

Figure 8. The effects of $p_{23,1}$ on the $R, v_{2(2)}$, and $v_{p(2)}$ for $\rho_{p}=1.0\left(R^{*}, v_{2(2)}^{*}\right.$, and $v_{(2)}^{*}$, respectively). Original polymer, Schulz-Zimm type distribution $\left(X_{w}^{0}=300\right.$, $\left.X_{w}^{0} / X_{n}^{0}=2\right) ; \chi_{12}^{0}=0.5, \chi_{13}^{0}=0.2$, and $\chi_{23}^{0}=1.0 . \quad p_{12,1}=$ $p_{13,1}=0, v_{p}^{0}=0.005$.
$X_{w} / X_{n}$ of the polymer in polymer-lean and rich phases $\left(X_{w(1)} / X_{n(1)}\right.$ and $\left.X_{w(2)} / X_{n(2)}\right)$ increases with an increase in $v_{p}^{0}$ and coincides with each other for $v_{p, c}^{0}$. As $\rho_{p}$ decreases from $1 / 15$ to zero, $v_{p, c}^{0}$ approaches to the critical polymer concentration.

Figure 10 shows the effects of $p_{12,1}, p_{13,1}$, and $p_{23,1}$ on the relation between the ratio $X_{w} / X_{n}$ of the two phases and $\rho_{p}$. In this figure the results ${ }^{14}$ for quasi-binary solutions with various $p_{1}$ are shown as broken lines for comparison. For any combination of $p_{12,1}$, $p_{13,1}$, and $p_{23,1}, X_{w} / X_{n}$ of the polymer in the polymer-lean phase $X_{w(1)} / X_{n(1)}$ decreases monotonically from 2.0 to 1.0 as an increase in $\rho_{p} . X_{w} / X_{n}$ of the polymer in a polymer-rich phase $X_{w(2)} / X_{n(2)}$ reveals minimum at a specific $\rho_{p}$, approaching to $X_{w}^{0} / X_{n}^{0}$ at $\rho_{p}=1.0$. Over a whole range of $\rho_{p} X_{w(2)} / X_{n(2)}$ attains minimum at $p_{12,1}=-0.2$ when $\chi_{12}^{0}=0.5, \chi_{13}^{0}=0.2$ and $\chi_{23}^{0}=1.0$ and becomes smaller for larger $p_{13,1}$ and $p_{23,1}$. Note that the relation between $X_{w(2)} / X_{n(2)}$ and $\rho_{p}$ for the quasiternary systems agrees with that of the quasibinary systems with $p_{1}=0.6$. For exam-

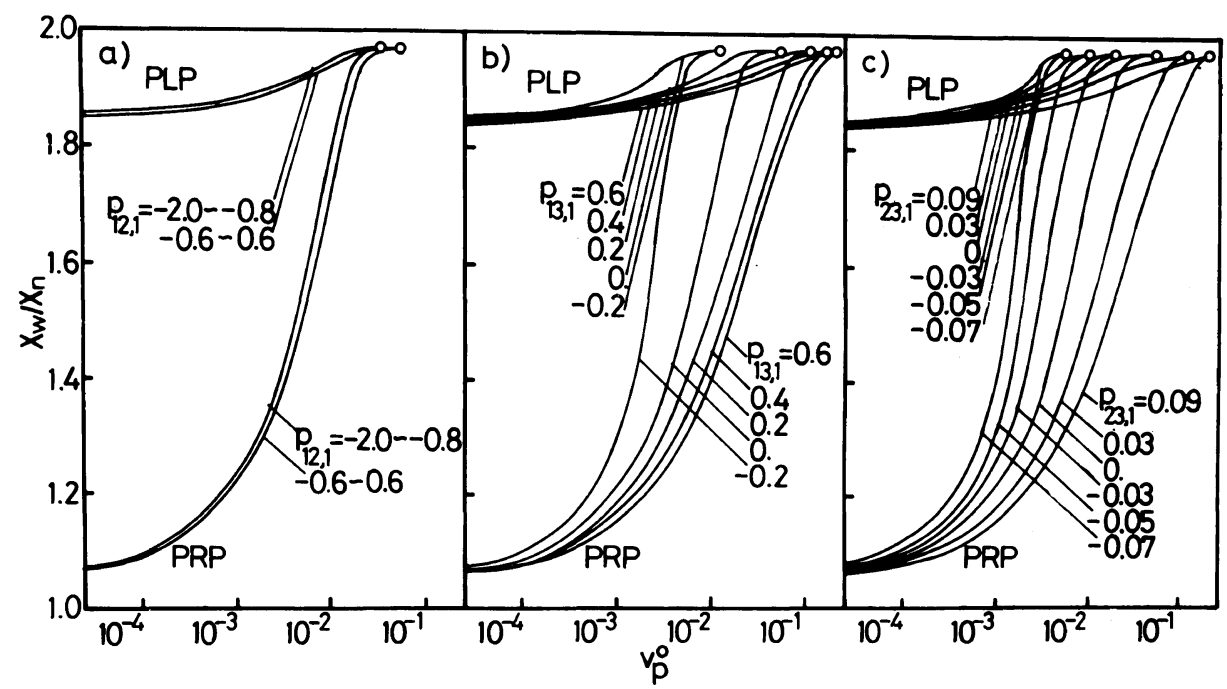

Figure 9. Effects of the concentration dependent parameters $p_{12,1}, p_{13,1}$, and $p_{23,1}$ on the relations between $X_{w} / X_{n}$ of the polymers in polymer-lean phase (PLP) or polymer-rich phase (PRP) and $v_{p}^{0}$. Original polymer, Schulz-Zimm type distribution $\left(X_{w}^{0}=300, X_{w}^{0} / X_{n}^{0}=2\right) ; \chi_{12}^{0}=0.5, \chi_{13}^{0}=0.2$, and $\chi_{23}^{0}=1.0, \rho_{p}=$ 1/15. a) $p_{13,1}=p_{23,1}=0$, b) $p_{12,1}=p_{23,1}=0$, c) $p_{12,1}=p_{13,1}=0$. Unfilled circle denotes $v_{p, c}^{0}$. 
ple, when $p_{13,1}=0.6 \quad\left(p_{12,1}=p_{23,1}=0\right) \quad$ or $p_{23,1}=0.05\left(p_{12,1}=p_{13,1}=0\right)$ for the quasiternary systems (SZ polymer, $X_{w}^{0}=300$, $\left.X_{w}^{0} / X_{n}^{0}=2, v_{p}^{0}=0.005\right), X_{w(2)} / X_{n(2)}$ vs. $\rho_{p}$ relation almost coincides with that for the quasibinary systems (SZ polymer, $X_{w}^{0}=300$, $\left.X_{w}^{0} / X_{n}^{0}=2, v_{p}^{0}=0.005\right)$. In a previous paper, ${ }^{9}$ we demonstrated that simply by a proper choice of $\chi_{12}^{0}, \chi_{13}^{0}$, and $\chi_{23}^{0}$ values, polymers could not be prepared having narrower MWD than those obtained from a quasibinary solution with $p_{1}=0$. Combination of solvents 1 and 2 with larger values of $p_{13,1}$ and $p_{23,1}$ (in this case, $p_{13,1} \geqq 0.6$ and $p_{23,1} \geqq$ 0.05 , respectively) will afford us polymer having narrower MWD than that obtained from quasi-binary system in a polymer fractionation experiment. It has been shown that the effects of $\chi_{13}^{0}$ on $g_{(2)}\left(X_{i}\right)$ is small but $\chi_{23}^{0}$ has a large effect on $g_{(2)}\left(X_{i}\right){ }^{10}$ Figure 10 in- dicates that $p_{23,1}$ has a strong influence on $g_{(2)}\left(X_{i}\right)$, accordingly $X_{w(2)} / X_{w(2)}$. In other words, a suitable choice of solvent 2 (i.e., non-solvent) is required to separate the polymers with sharp MWD from quasi-ternary systems.

Figure 11 shows the effects of $p_{12,1}, p_{13,1}$, and $p_{23,1}$ on the relation between the standard deviation of MWD $\sigma^{\prime} \quad\left(\equiv\left\{X_{w}\left(X_{z}-\right.\right.\right.$ $\left.\left.X_{w}\right)\right\}^{1 / 2}, X_{z}$, the $z$-average of $\left.X_{i}\right)$ of the polymers partitioned in two phases and $v_{p}^{0}$ in the case of $\rho_{p}=1 / 15$. Broken line in the figure shows the $\sigma^{\prime}$ of original polymer $\left[\sigma_{0}^{\prime}\right.$ $(=212.1)]$. In the figure unfilled circle is the $v_{p, c}^{0} . \sigma^{\prime}$ of the polymer in PLP $\left(\sigma_{(1)}^{\prime}\right)$ increases with an increase in $v_{p}^{0}$ and almost coincides with $\sigma_{0}^{\prime}$ at $v_{p, c}^{0}$, which approaches to $v_{p}^{c}$ for $\rho_{p} \rightarrow 0 . X_{n(1)}, X_{w(1)}$, and $X_{z(1)}$ increase with an increase in $v_{p}^{0} . \sigma_{(1)}^{\prime}$ is very close to $\sigma_{0}^{\prime}$ at $v_{p}^{0}=v_{p, c}^{0}$. On the other hand, $X_{n(2)}, X_{w(2)}$, and

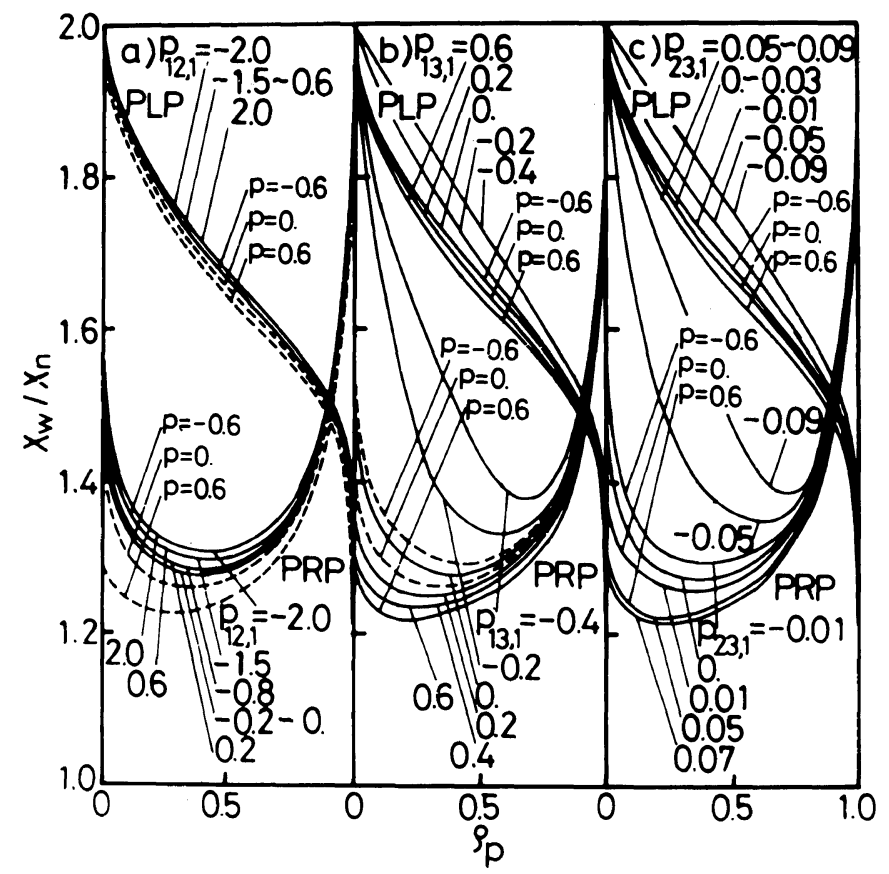

Figure 10. Effects of the concentration dependent parameters $p_{12,1}, p_{13,1}$, and $p_{23,1}$ on the relations between $X_{w} / X_{n}$ of the polymers in polymer-lean phase (PLP) or polymer-rich phase (PRP) and $\rho_{p}$. Original polymer, Schulz-Zimm type distribution $\left(X_{w}^{0}=300, X_{w}^{0} / X_{n}^{0}=2\right) ; \chi_{12}^{0}=0.5, \chi_{13}^{0}=0.2$, and $\chi_{23}^{0}=1.0, v_{p}^{0}=$ 0.005. a) $p_{13,1}=p_{23,1}=0$, b) $p_{12,1}=p_{23,1}=0$, c) $p_{12,1}=p_{13,1}=0$. The broken lines are the results for quasibinary system. $p$ is denoted on curve. 




Figure 11. Effects of the concentration dependent parameters $p_{12,1}, p_{13,1}$, and $p_{23,1}$ on the relations between $\sigma^{\prime}$ of the polymers in polymer-lean phase (PLP) or polymer-rich phase (PRP) and $v_{p}^{0}$. Original polymer, Schulz-Zimm type distribution $\left(X_{w}^{0}=300, X_{w}^{0} / X_{n}^{0}=2\right) ; \chi_{12}^{0}=0.5, \chi_{13}^{0}=0.2$, and $\chi_{23}^{0}=1.0, \rho_{p}=$ 1/15. a) $p_{13,1}=p_{23,1}=0$, b) $p_{12,1}=p_{23,1}=0$, c) $p_{12,1}=p_{13,1}=0$. Unfilled circle denotes $v_{p, c}^{0}$.



Figure 12. Effects of the concentration dependent parameters $p_{12,1}, p_{13,1}$, and $p_{23,1}$ on the relations between $\sigma^{\prime}$ of the polymers in polymer-lean phase (PLP) or polymer-rich phase (PRP) and $\rho_{p}$. Original polymer, Schulz-Zimm type distribution $\left(X_{w}^{0}=300, X_{w}^{0} / X_{n}^{0}=2\right) ; \chi_{12}^{0}=0.5, \chi_{13}^{0}=0.2$, and $\chi_{23}^{0}=1.0, v_{p}^{0}=$ 0.005 . a) $p_{13,1}=p_{23,1}=0$, b) $p_{12,1}=p_{23,1}=0$, c) $p_{12,1}=p_{13,1}=0$. The broken lines are the results for quasibinary system. $p$ is denoted on curve.

$X_{z(2)}$ decrease and $X_{z(2)}-X_{w(2)}$ increases monotonically as $v_{p}^{0}$ increases. In the range of $v_{p}^{0} \leqq 1 \cdot 10^{-4}, \sigma_{(2)}^{\prime}$ is smaller than $\sigma_{0}^{\prime}$. With an increase in $X_{z(2)}-X_{w(2)}, \sigma_{(2)}^{\prime}$ gradually increases and coincides with $\sigma_{0}^{\prime}$ at $v_{p}^{0} \sim 1 \times$ $10^{4}$. In spite of the agreement of $\sigma_{(2)}^{\prime}$ with $\sigma_{0}^{\prime}$ 


\section{S. MATSUDA}

at $v_{p}^{0} \sim 1 \times 10^{-4}$, neither $X_{w(2)}=X_{w}^{0}$ nor $X_{z(2)}=X_{z}^{0}$ hold even at $v_{p}^{0} \sim 1 \times 10^{-4}$ but $X_{w(2)} \gg X_{w}^{0}$ and $X_{z(2)} \gg X_{z}^{0}$ hold. $\sigma_{(2)}^{\prime}$ attains a maximum at specific $v_{p}^{0}\left(\equiv v_{p}^{0}\right.$, max $)$ because the effect of decrease in $X_{w(2)}$ becomes larger than those of increase in $X_{z(2)}-X_{w(2)}$. Fur-

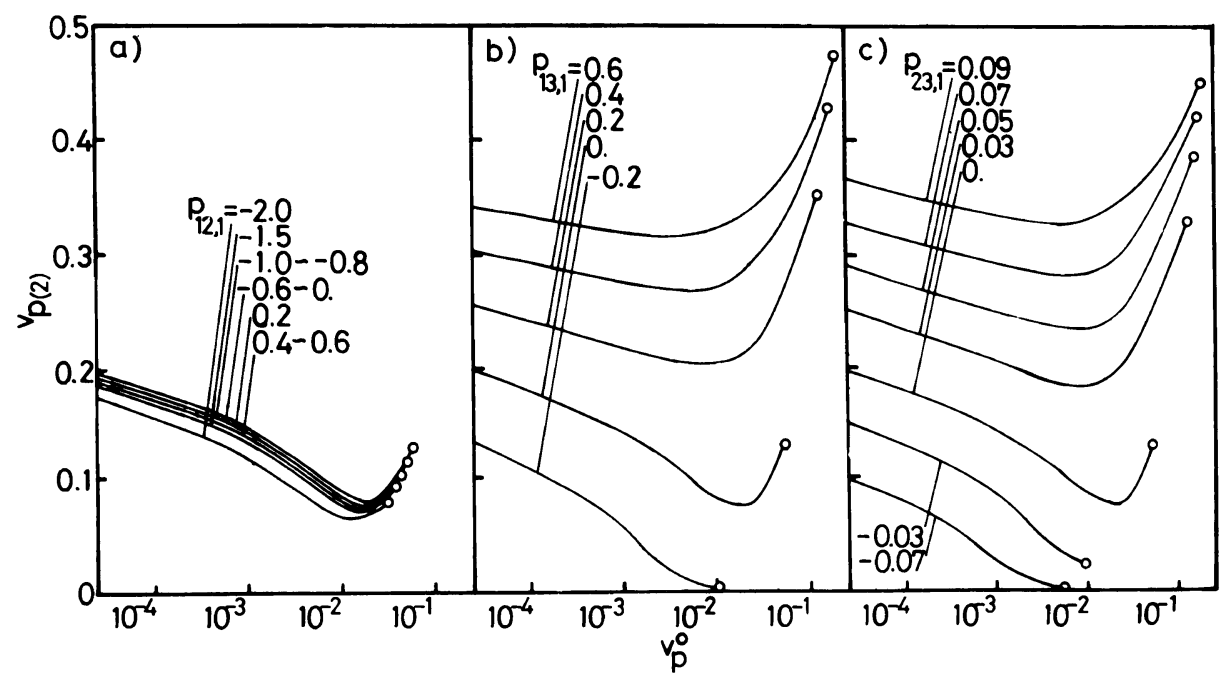

Figure 13. Effects of the concentration dependent parameters $p_{12,1}, p_{13,1}$, and $p_{23,1}$ on the relations between polymer volume fraction in polymer-rich phase $v_{p(2)}$ and $v_{p}^{0}$. Original polymer, Schulz-Zimm type distribution $\left(X_{w}^{0}=300, X_{w}^{0} / X_{n}^{0}=2\right) ; \chi_{12}^{0}=0.5, \chi_{13}^{0}=0.2$, and $\chi_{23}^{0}=1.0, \rho_{p}=1 / 15$. a) $p_{13,1}=p_{23,1}=0$, b) $p_{12,1}=p_{23,1}=0$, c) $p_{12,1}=p_{13,1}=0$. Unfilled circle denotes $v_{p, c^{*}}^{0}$.

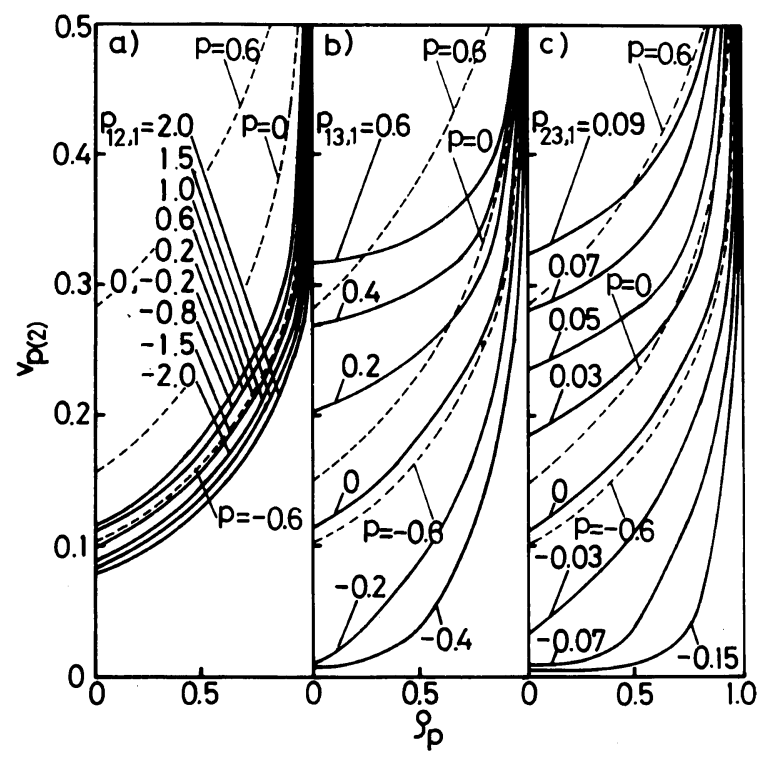

Figure 14. Effects of the concentration dependent parameters $p_{12,1}, p_{13,1}$, and $p_{23,1}$ on the relations between polymer volume fraction in polymer-rich phase $v_{p(2)}$ and $\rho_{p}$. Original polymer, Schulz-Zimm type distribution $\left(X_{w}^{0}=300, X_{w}^{0} / X_{n}^{0}=2\right) ; \chi_{12}^{0}=0.5, \chi_{13}^{0}=0.2$, and $\chi_{23}^{0}=1.0, v_{p}^{0}=0.005$. a) $p_{13,1}=p_{23,1}=0$, b) $p_{12,1}=p_{23,1}=0$, c) $p_{12,1}=p_{13,1}=0$. The broken lines are the results for quasi-binary system. $p$ is denoted on curve. 
ther, $\sigma_{(2)}^{\prime}$ rapidly decreases with an increase in $v_{p}^{0}$. When $v_{p}^{0}=v_{p, c}^{c}, X_{n(2)}, X_{w(2)}$, and $X_{z(2)}$ are fairly larger than $X_{n}^{0}, X_{w}^{0}$, and $X_{z}^{0}$, respectively and $\sigma_{(2)}^{\prime}$ is larger than $\sigma_{0}^{\prime}$. At $v_{p}^{0}=v_{p, c}^{0}$, $X_{w(2)} / X_{n(2)}$ coincides with $X_{w(1)} / X_{n(1)}$ as shown in Figure 9, but note that $X_{n(2)} \gg$ $X_{n(1)}$ and $X_{w(2)} \gg X_{n(1)}$ always hold. As $v_{p, c}^{0}$ increases with an increase in $p_{13,1}$ and $p_{23,1}$, we can predict that $v_{p}^{c}$ slowly increases with an increase in $p_{13,1}$ and $p_{23,1}$.

Figure 12 shows the effects of $p_{12,1}, p_{13,1}$, and $p_{23,1}$ on $\sigma^{\prime}$ vs. $\rho_{p}$ curve. The figure also includes the results ${ }^{20-27}$ of quasi-binary system as broken line for comparison. Filled circle denotes the value for the original polymer $\left(\sigma_{0}^{\prime}\right)$. As well as $X_{w(2)} / X_{n(2)}$ vs. $\rho_{p}$ curve, $\sigma_{(2)}^{\prime} v s . \rho_{p}$ curve has a minimum at some $\rho_{p} . \sigma_{(1)}^{\prime}$ and $\sigma_{(2)}^{\prime}$ gradually decrease with an increase in $p_{13,1}$ and $p_{23,1} . \sigma_{(2)}^{\prime}$ of the quasiternary system with $p_{13,1}=0.4 \sim 0.6\left(p_{12,1}=\right.$ $\left.p_{23,1}=0\right)$ or $p_{23,1}=0.05 \sim 0.09\left(p_{12,1}=p_{13,1}=\right.$ $0)$ agrees fairly with $\sigma_{(2)}^{\prime}$ of the quasi-binary system with $p_{1}=0.6$. Similar behavior is observed for $\sigma_{(1)}^{\prime}$.

Figures 13 and 14 show the effects of $p_{12,1}$, $p_{13,1}$, and $p_{23,1}$ on the $v_{p(2)} v s, v_{p}^{0}$ curve and $v_{p(2)} v s . \rho_{p}$ curve, respectively. The effect of $p_{12,1}$ on $v_{p(2)}$ is small but innegligible and $v_{p(2)}$ for a given $\rho_{p}$ shows a maximum at $p_{12,1}$, ranging between -0.6 and 0 . The effects of $p_{13,1}$ and $p_{23,1}$ are remarkable and $v_{p(2)}$ increases abruptly with an increase in $\rho_{13,1}$ and $\rho_{23,1}$. Especially, $v_{p(2)}$ increases suddenly with a small increase in $p_{23,1} . v_{p(2)} v s . v_{p}^{0}$ curve has a minimum at the point corresponding to the maximum of $V_{(2)}$ vs. $v_{p}^{0}$ curve. $v_{p(2)}$ increases with an increase in $\rho_{p}$, and coincides with $v_{p(2)}^{*}$ at $\rho_{p}=1$ (see Figure 8). By use of eq $\mathrm{C}-8 \mathrm{~b}$, relation between $R$ and $\rho_{p}$ is expressed by:

$$
R=\frac{v_{p(2)}}{v_{p}^{0} \rho_{p}}-1
$$

In the range of the small $\rho_{p}\left(i, e ., \rho_{p}<0.3\right)$ ), $R$ decreases abruptly with an increase in $\rho_{p}$ because $v_{p(2)}$ changes slowly with $\rho_{p}$. In con- trast to this, in the large $\rho_{p}$ region $R$ increases gradually with $\rho_{p}$ because of the rapid change of $v_{p(2)}$ with $\rho_{p}$.

Equation C-8a can be rewritten as follows.



Figure 15. Effects of $p_{12,1}, p_{13,1}$, and $p_{23,1}$ on $v_{1(1)}, v_{2(1)}$, $v_{1(2)}$, and $v_{2(2)} v s$. $\rho_{p}$ curves. Original polymer, SchulzZimm type distribution $\left(X_{w}^{0}=300, X_{w}^{0} / X_{n}^{0}=2\right) ; \chi_{12}^{0}=0.5$, $\chi_{13}^{0}=0.2$, and $\chi_{23}^{0}=1.0, v_{p}^{0}=0.005$. a) and d) $p_{13,1}=$ $p_{23,1}=0$, b) and e) $p_{12,1}=p_{23,1}=0$, c) and f) $p_{12,1}=$ $p_{13,1}=0$. The broken lines are the results for quasibinary system. $p$ is denoted on curve. 


$$
v_{p(1)}=v_{p}^{0} \rho_{s}\left(1+\frac{1}{R}\right)
$$

If $1 / R \ll 1, v_{p(1)}$ is independent of $\chi_{12}^{0}, \chi_{13}^{0}, \chi_{23}^{0}$, $p_{12, s}, p_{13, q}$, and $p_{23, r}$ and dependent on $\rho_{p}$ and $v_{p}^{0}$ (see for example, Figure 19 of ref 10 ).

The effects of $p_{12,1}$, and $p_{13,1}$, and $p_{23,1}$ on $v_{1(1)}, v_{2(1)}, v_{1(2)}$, and $v_{2(2)}$ vs. $\rho_{p}$ curves are shown in Figure 15. Generally, with $a$ decrease in $p_{12,1}$ and an increase in $p_{13,1}$ and $p_{23,1}, v_{1(1)}, v_{1(2)}$, and $v_{2(2)}$ decrease and $v_{2(1)}$ increases. The effect of concentration dependence of $\chi$-parameters increases in the following order: $p_{12,1}<p_{13,1}<p_{23,1}$. The effect of $p_{12,1}$ on $v_{1(2)}$ is just reverse to that on $v_{2(2)}$ and $v_{1(2)}+v_{2(2)}$ is insignificantly influenced by $p_{12,1} \cdot v_{1(1)}\left(\right.$ or $\left.v_{1(2)}\right) v s$. $\rho_{p}$ curve is similar to $V_{1}$ $v s$. $\rho_{p}$ curve in shape and $v_{2(1)} v s . \rho_{p}$ curve has a similar shape to $V_{2}$ vs. $\rho_{p}$ curve. As $\rho_{p}$ increases, $v_{1(1)}$ and $v_{1(2)}$ decrease rapidly, approaching to zero at $\rho_{p}=1.0$ and $v_{2(1)}$ increases remarkably, approaching to unity at $\rho_{p}=1.0$. Extrapolated value of $v_{2(2)}$ to $\rho_{p}=1$ is denoted by filled circle, which coincides with $v_{2(2)}^{*}$ calculated by eq 52 .
Figure 16 shows the effects of $p_{12,1}, p_{13,1}$, and $p_{23,1}$ on the relation between $V$ (or $V_{2}$ ) and $\rho_{p}$ when solvent 2 (poor solvent) was added to the starting solution consisting of solvent 1 (good solvent) and polymer with $v_{p}^{s}=$ 0.01 . As $p_{12,1}$ decreases and $p_{13,1}$, and $p_{23,1}$ increase, a large amount of solvent 2 is needed to bring about the two phase separation. Especially small change in $p_{23,1}$ causes a drastic change in $V_{2}$. Considering the strong effects of $p_{23,1}$ and $\chi_{23}^{0}$ on $V_{2}$ as first demonstrated by Kamide and Matsuda, ${ }^{10}$ a careful choice of non-solvent is an important factor to determine $V_{2}$ (or $V$ ).

Figure 17 shows the effect of the concentration dependence of three thermodynamic interaction parameters on the coexisting curve. Phase separation is obtained under the same conditions as those in Figure 16. In the figure tie lines for $\rho_{p}=1 / 100$ are shown by the full lines, which can be accurately approximated with tie line for $\rho_{p}=0$ (limiting tie-line) and the line of $v_{p}^{s}=0.01$ is shown by the broken line. The cross point of the limiting tie-line and the line of $v_{p}^{s}=0.01$ can be

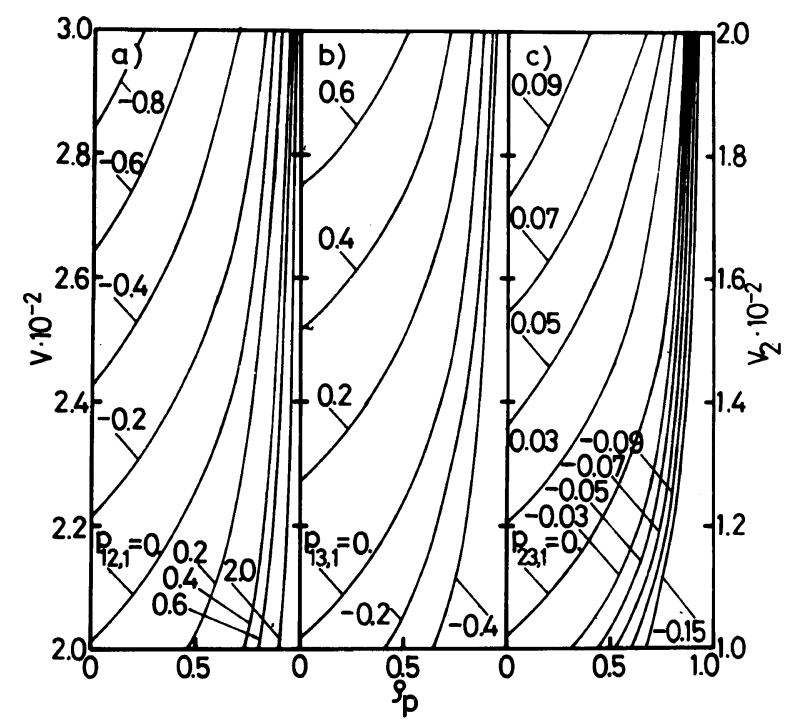

Figure 16. Effects of the concentration dependent parameters $p_{12,1}, p_{13,1}$, and $p_{23,1}$ on the relations between the total volume of the system $V$ (or volume of nonsolvent $V_{2}$ ) and $\rho_{p}$. Original polymer, SchulzZimm type distribution $\left(X_{w}^{0}=300, X_{w}^{0} / X_{n}^{0}=2\right) ; \chi_{12}^{0}=0.5, \chi_{13}^{0}=0.2$, and $\chi_{23}^{0}=1.0, v_{p}^{s}=0.01$. a) $p_{13,1}=$ $p_{23,1}=0$, b) $p_{12,1}=p_{23,1}=0$, c) $p_{12,1}=p_{13,1}=0$. 
Phase Equilibria of Quasi-Ternary Systems V.

regarded as a cloud point. In the previous $\chi_{12}^{0}$ and a increase in $\chi_{13}^{0}$ and $\chi_{23}^{0}$. With an paper, ${ }^{9}$ the coexisting curve shifted to a di- increase in $p_{12,1}$ from -2.0 to 0.6 , a similar rection of decreasing $v_{2}$ with a decrease in shift in the coexisting curve was observed, but
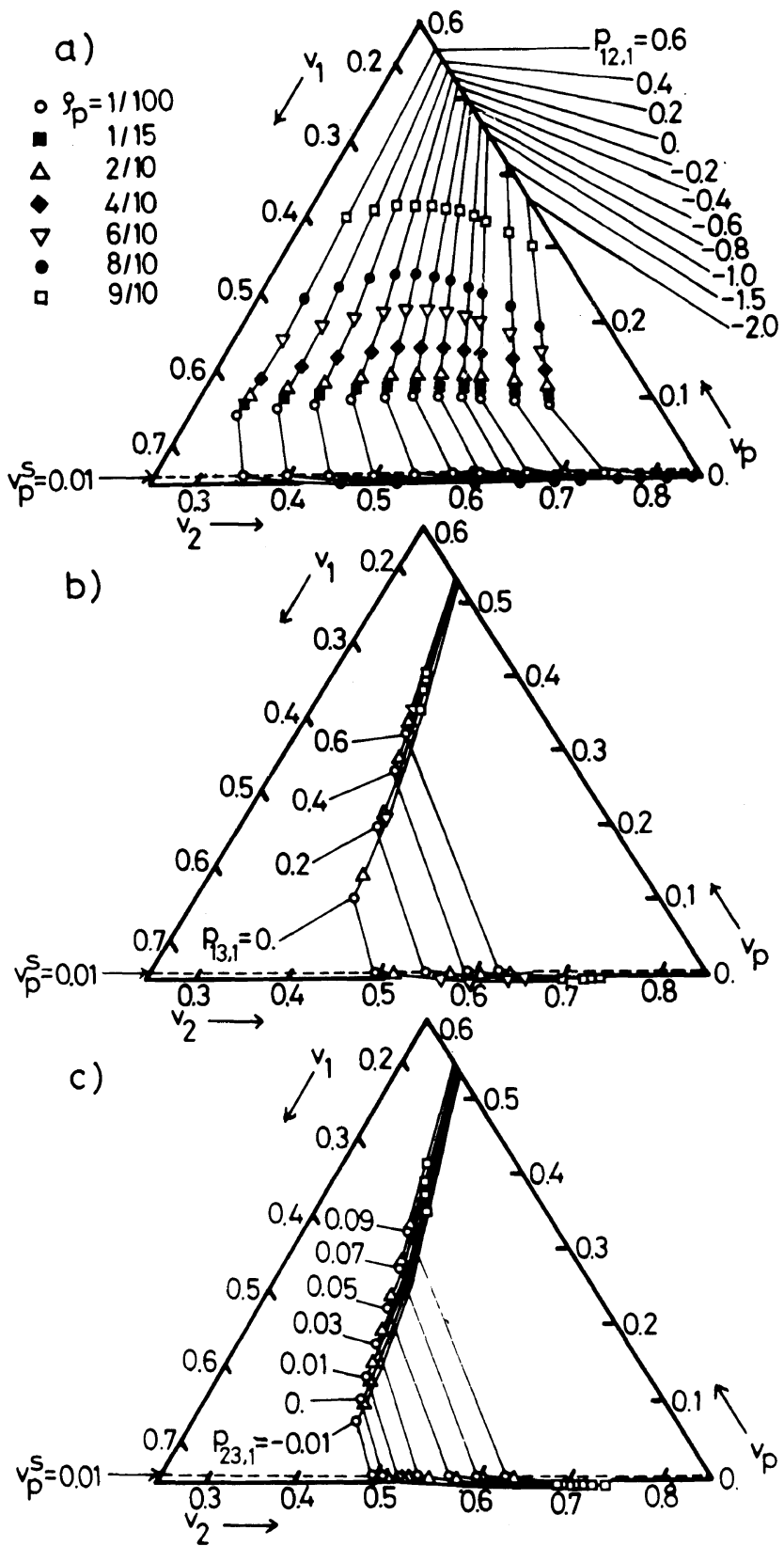

Figure 17. Effects of the concentration dependent parameters $p_{12,1}, p_{13,1}$, and $p_{23,1}$ on the coexisting curves and their tie-lines for quasi-ternary system consisting of multicomponent polymer (Schulz-Zimm distribution, $X_{w}^{0}=300, X_{w}^{0} / X_{n}^{0}=2$ ) in binary solvent mixture. $\chi_{12}^{0}=0.5, \chi_{13}^{0}=0.2$, and $\chi_{23}^{0}=1.0, v_{p}^{s}=0.01$. a) $p_{13,1}=p_{23,1}=0$, b) $p_{12,1}=p_{23,1}=0$, c) $p_{12,1}=p_{13,1}=0$. $\bigcirc, \rho_{p}=1 / 100 ; \boldsymbol{\square}, \rho_{p}=1 / 15 ; \Delta, \rho_{p}=2 / 10 ; \bullet, \rho_{p}=$ $4 / 10 ; \nabla, \rho_{p}=6 / 10 ; 0, \rho_{p}=8 / 10 ; \square, \rho_{p}=9 / 10$. 


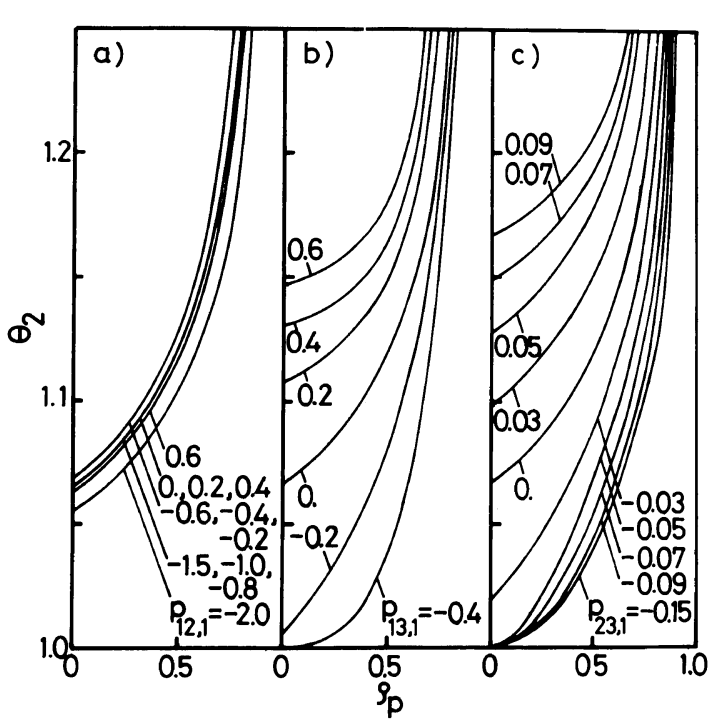

Figure 18. Effects of the concentration dependent parameters $p_{12,1}, p_{13,1}$, and $p_{23,1}$ on the relations between degree of selective adsorption $\theta_{2}$ and $\rho_{p}$. Original polymer, Schulz-Zimm type distribution $\left(X_{p}^{0}=300\right.$, $\left.X_{w}^{0} / X_{n}^{0}=2\right) ; \chi_{12}^{0}=0.5, \chi_{13}^{0}=0.2$, and $\chi_{23}^{0}=1.0, v_{p}^{s}=0.01$. a) $p_{13,1}=p_{23,1}=0$, b) $p_{12,1}=p_{23,1}=0$, c) $p_{12,1}=p_{13,1}=0$.

$v_{p(2)}$ changes a little, showing a maximum at $p_{12,1}=-0.2$. With an increase in $p_{13,1}$ and $p_{23,1}$, limiting tie-line approaches to $v_{2}-v_{p}$ axis and $v_{p(2)}$ increases drastically.

Figure 18 shows the effects of $p_{12,1}, p_{13,1}$, and $p_{23,1}$ on the relation between $\theta_{2}$, which is defined by following equation,

$$
\theta_{2}=\frac{v_{2(1)} /\left(v_{1(1)}+v_{2(1)}\right)}{v_{2(2)} /\left(v_{1(2)}+v_{2(2)}\right)}
$$

and $\rho_{p}$. As $\theta_{2}$ is always larger than 1.0, a larger amount of non-solvent is partitioned in polymer-lean phase than - rich phase. The fact that $\theta$ increases with an increase in $\rho_{p}$ suggests strongly that tie-line changes from $v_{1}-v_{p}$ axis parallel to $v_{2}-v_{p}$ axis parallel.

Summarizing, (a) a good separation efficiency can be expected for (i) small $\chi_{12}^{0}$ and $p_{12,1} \sim-0.2$, (ii) large $\chi_{13}^{0}$ and large $p_{13,1}$, (iii) small $\chi_{23}^{0}$ and large $p_{23,1}$. Specially, careful choice of solvent 2 (non-solvent) is important. (b) For large $p_{13,1}$ and $p_{23,1}$, separation efficiency in quasi-ternary system exceeds that in quasi-binary system. (c) In order to diminish the amount of non-solvent necessary to separate the polymer in the polymerrich phase at the same $\rho_{p}$ (i) small $\chi_{12}^{0}$ and large $p_{12,1}$, (ii) large $\chi_{13}^{0}$ and small $p_{13,1}$, (iii) large $\chi_{23}^{0}$ and small $p_{23,1}$ are desired.

Acknowledgements. The author should like to express his most sincere gratitude to Dr. Kenji Kamide, Director of Fundamental Research Laboratory of Fibers and Fiber-Forming Polymers, Asahi Chemical Industry Co., Ltd., for his guidance.

\section{APPENDIX A}

Derivation of eq 13-15.

Integrating eq $9, \Delta G^{E}$ for quasi-binary system is obtained: ${ }^{14}$

$$
\begin{aligned}
\Delta G^{E}= & \int_{0}^{N_{0}}\left(\frac{\partial \Delta G^{E}}{\partial N_{0}}\right) \mathrm{d} N_{0} \\
= & \tilde{R} T\left(N_{0}+\sum_{i=1}^{m} X_{i} N_{X_{i}}\right) \\
& \times\left[\chi_{0}\left(1+\sum_{j=1}^{n} \frac{p_{j}}{j+1} \frac{1-v_{p}^{j+1}}{v_{0}}\right)\right] v_{0} v_{p}
\end{aligned}
$$

Following the procedure of quasi-binary system, $\Delta G_{13}^{E}$ and $\Delta G_{23}^{E}$ for quasi-ternary system can be given by integration of eq 10 and 11 , respectively.

$$
\begin{aligned}
\Delta G_{13}^{E}= & \int_{0}^{N_{1}}\left(\frac{\partial \Delta G_{13}^{E}}{\partial N_{1}}\right) \mathrm{d} N_{1} \\
= & \tilde{R} T\left(N_{1}+N_{2}+\sum_{i=1}^{m} X_{i} N_{X_{i}}\right) \\
& \times\left[\chi _ { 1 3 } ^ { 0 } \left\{1+\sum_{q=1}^{n_{q}} \frac{p_{13, q}}{q+1}\left(\frac{v_{p}}{v_{2}+v_{p}}\right)^{q}\right.\right. \\
& \left.\left.\times \frac{1-\left(v_{2}+v_{p}\right)^{q+1}}{v_{1}}\right\}\right] v_{1} v_{p} \\
\Delta G_{23}^{E}= & \int_{0}^{N_{2}}\left(\frac{\partial \Delta G_{23}^{E}}{\partial N_{2}}\right) \mathrm{d} N_{2}
\end{aligned}
$$




$$
\begin{aligned}
= & \tilde{R} T\left(N_{1}+N_{2}+\sum_{i=1}^{m} X_{i} N_{X_{i}}\right) \\
& \times\left[\chi_{23}^{0}\left\{1+\sum_{r=1}^{n_{r}} \frac{p_{23, r}}{r+1}\right)\left(\frac{v_{p}}{v_{1}+v_{p}}\right)^{r}\right. \\
& \left.\left.\times \frac{1-\left(v_{1}+v_{p}\right)^{r+1}}{v_{2}}\right\}\right] v_{2} v_{p}
\end{aligned}
$$

$\Delta G^{i d}$ is expressed by eq A-4. ${ }^{15}$

$$
\begin{aligned}
\Delta G^{i d}= & \tilde{R} T\left(N_{1}+N_{2}+\sum_{i=1}^{m} X_{i} N_{X_{i}}\right) \\
& \times\left[v_{1} \ln v_{1}+v_{2} \ln v_{2}+\sum_{i=1}^{m} v_{X_{i}} \ln v_{X_{i}}\right]
\end{aligned}
$$

Substituting eq 12 , and A-2 $\sim \mathrm{A}-4$ into eq $8, \Delta G$ is given by eq $\mathrm{A}-5$

$$
\begin{aligned}
\Delta G= & \tilde{R} T\left(N_{1}+N_{2}+\sum_{i=1}^{m} X_{i} N_{X_{i}}\right) \\
& \times\left[v_{1} \ln v_{1}+v_{2} \ln v_{2}+\sum_{i=1}^{m} v_{X_{i}} \ln v_{X_{i}}\right. \\
& +\chi_{12}^{0}\left(1+\sum_{s=1}^{n_{s}} p_{12, s} v_{p}^{s}\right) v_{1} v_{2} \\
& +\chi_{13}^{0}\left\{1+\sum_{q=1}^{n_{q}} \frac{p_{13, q}}{q+1}\left(\frac{v_{p}}{v_{2}+v_{p}}\right)^{q}\right. \\
& \left.\times \frac{1-\left(v_{2}+v_{p}\right)^{q+1}}{v_{1}}\right\} v_{1} v_{p} \\
& +\chi_{23}^{0}\left\{1+\sum_{r=1}^{n_{r}} \frac{p_{23, r}}{r+1}\left(\frac{v_{p}}{v_{1}+v_{p}}\right)^{r}\right. \\
& \left.\left.\times \frac{1-\left(v_{1}+v_{p}\right)^{r+1}}{v_{2}}\right\} v_{2} v_{p}\right]
\end{aligned}
$$

Differentiations of $\Delta G$ by $N_{1}, N_{2}$, and $N_{X_{i}}$ give $\Delta \mu_{1}, \Delta \mu_{2}$, and $\Delta \mu_{X_{i}}(i=1, \cdots, m)$ as eq 13 , 14 , and 15 , respectively.

$\Delta G^{i d}, \Delta G_{12}^{E}, \Delta G_{13}^{E}$, and $\Delta G_{23}^{E}$ satisfy following relations,

$$
N_{1} \mathrm{~d}\left(\frac{\partial \Delta G^{i d}}{\partial N_{1}}\right)+N_{2} \mathrm{~d}\left(\frac{\partial \Delta G^{i d}}{\partial N_{2}}\right)
$$

$$
\begin{array}{r}
+\sum_{i=1}^{m} N_{X_{i}} \mathrm{~d}\left(\frac{\partial \Delta G^{i d}}{\partial N_{X_{i}}}\right)=0 \\
N_{1} \mathrm{~d}\left(\frac{\partial \Delta G_{12}^{E}}{\partial N_{1}}\right)+N_{2} \mathrm{~d}\left(\frac{\partial \Delta G_{12}^{E}}{\partial N_{2}}\right) \\
+\sum_{i=1}^{m} N_{X_{i}} \mathrm{~d}\left(\frac{\partial \Delta G_{12}^{E}}{\partial N_{X_{i}}}\right)=0 \\
N_{1} \mathrm{~d}\left(\frac{\partial \Delta G_{13}^{E}}{\partial N_{1}}\right)+N_{2} \mathrm{~d}\left(\frac{\partial \Delta G_{13}^{E}}{\partial N_{2}}\right) \\
+\sum_{i=1}^{m} N_{X_{i}} \mathrm{~d}\left(\frac{\partial \Delta G_{13}^{E}}{\partial N_{X_{i}}}\right)=0 \\
N_{1} \mathrm{~d}\left(\frac{\partial \Delta G_{23}^{E}}{\partial N_{1}}\right)+N_{2} \mathrm{~d}\left(\frac{\partial \Delta G_{23}^{E}}{\partial N_{2}}\right) \\
+\sum_{i=1}^{m} N_{X_{i}} \mathrm{~d}\left(\frac{\partial \Delta G_{23}^{E}}{\partial N_{X_{i}}}\right)=0
\end{array}
$$

namely, Gibbs-Duhem relation holds among $\Delta \mu_{1}, \Delta \mu_{2}$, and $\Delta \mu_{X_{i}}(i=1, \cdots, m)$.

$$
N_{1} \mathrm{~d}\left(\Delta \mu_{1}\right)+N_{2} \mathrm{~d}\left(\Delta \mu_{2}\right)+\sum_{i=1}^{m} N_{X_{i}} \mathrm{~d}\left(\Delta \mu_{X_{i}}\right)=0
$$

Equation A-10 can be converted into eq A-11:

$$
\begin{aligned}
N_{1}\left[\left(\frac{\partial \Delta \mu_{1}}{\partial v_{1}}\right) \mathrm{d} v_{1}+\left(\frac{\partial \Delta \mu_{1}}{\partial v_{p}}\right) \mathrm{d} v_{p}\right. \\
\left.\quad+\left(\frac{\partial \Delta \mu_{1}}{\partial\left(1 / X_{n}\right)}\right) \mathrm{d}\left(1 / X_{n}\right)\right] \\
\quad+N_{2}\left[\left(\frac{\partial \Delta \mu_{2}}{\partial v_{1}}\right) \mathrm{d} v_{1}+\left(\frac{\partial \Delta \mu_{2}}{\partial v_{p}}\right) \mathrm{d} v_{p}\right. \\
\left.\quad+\left(\frac{\partial \Delta \mu_{2}}{\partial\left(1 / X_{n}\right)}\right) \mathrm{d}\left(1 / X_{n}\right)\right] \\
+\sum_{i=1}^{m} N_{X_{i}}\left[\left(\frac{\partial \Delta \mu_{X_{i}}}{\partial v_{1}}\right) \mathrm{d} v_{1}\right. \\
+\left(\frac{\partial \Delta \mu_{X_{i}}}{\partial v_{p}}\right) \mathrm{d} v_{p}+\left(\frac{\partial \Delta \mu_{X_{i}}}{\partial v_{X_{i}}}\right) \mathrm{d} v_{X_{i}} \\
\left.+\left(\frac{\partial \Delta \mu_{X_{i}}}{\partial\left(1 / X_{n}\right)}\right) \mathrm{d}\left(1 / X_{n}\right)\right]=0
\end{aligned}
$$


Substituting eq $13-15$ into A-11, we can readily confirm eq A-10.

\section{APPENDIX B}

Relation between $\chi$ and $g$

In the case of quasi-binary system, following relation holds between $\chi$ (given by eq 4) and $g$ (introduced by Koningsveld et al. $\left.^{16,17}\right):^{15}$

$$
\begin{aligned}
g & =\frac{1}{v_{0}} \int_{1-v_{0}}^{1} \chi \mathrm{d}\left(1-v_{0}\right) \\
& =\chi_{0}\left[1+\sum_{j=1}^{n} \frac{p_{j}}{j+1} \frac{1-v_{p}^{j+1}}{v_{0}}\right]
\end{aligned}
$$

Differential form of the relation between $\chi$ and $g$ is

$$
\chi=g-v_{0} \frac{\partial g}{\partial\left(1-v_{0}\right)}
$$

where $v_{0}\left(=1-v_{p}\right)$ is volume fraction of solvent. Equation B-2 is consistent with eq B-1. For quasi-ternary system combination of eq 17 and A-2 gives relation between $g_{13}$ and $\chi_{13}$,

$$
\begin{aligned}
g_{13}= & \chi_{13}^{0}\left\{1+\sum_{q=1}^{n_{q}} \frac{p_{13, q}}{q+1}\left(\frac{v_{p}}{v_{2}+v_{p}}\right)^{q}\right. \\
& \left.\times \frac{1-\left(v_{2}+v_{p}\right)^{q+1}}{v_{1}}\right\} \\
= & \frac{\chi_{13}^{0}}{v_{1}}\left[\frac{N_{2}+\sum X_{i} N_{X_{i}}}{N_{1}+N_{2}+\sum X_{i} N_{X_{i}}}+\sum_{q=1}^{n_{q}} \frac{p_{13, q}}{q+1}\right. \\
& \left.\times \frac{\left(\sum X_{i} N_{X_{i}}\right)^{q}\left(N_{2}+\sum X_{i} N_{X_{i}}\right)}{\left(N_{1}+N_{2}+\sum X_{i} N_{X_{i}}\right)^{q+1}}\right]_{N_{1}=N_{1}}^{0} \\
= & \frac{\chi_{13}^{0}}{v_{1}} \int_{1-v_{1}}^{1}\left[1+\sum_{q=1}^{n_{q}} p_{13, q} v_{p}^{q}\right] \mathrm{d}\left(1-v_{1}\right)
\end{aligned}
$$

Equation B-3 is also rewritten in the differential form.

$$
g_{13}-v_{1} \frac{\partial g_{13}}{\partial\left(1-v_{1}\right)}=g_{13}-v_{1} \frac{\partial g_{13}}{\partial N_{1}} \frac{\partial N_{1}}{\partial\left(1-v_{1}\right)}
$$

$$
=\chi_{13}^{0}\left[1+\sum_{q=1}^{n_{q}} p_{13, q} v_{p}^{q}\right]=\chi_{13}
$$

According to the same procedure, relation between $g_{23}$ and $\chi_{23}$ is given by eq 21 and 23.

\section{APPENDIX C}

From the definition of $R$ and $V, V_{(1)}$ and $V_{(2)}$ are expressed by eq $\mathrm{C}-1 \mathrm{a}$ and $\mathrm{b}$.

$$
\begin{aligned}
& V_{(1)}=\frac{R}{R+1} V \\
& V_{(2)}=\frac{1}{R+1} V
\end{aligned}
$$

Substitution of eq C-1a and b into $V_{2}=$ $v_{2(1)} V_{(1)}+v_{2(2)} V_{(2)}$, yields,

$$
V_{2}=\frac{V}{R+1}\left(R v_{2(1)}+v_{2(2)}\right)
$$

and combination of eq $\mathrm{C}-2$ and 32 with $V=$ $V_{1}^{0}+V_{2}+V_{3}^{0}$ gives:

$$
\begin{gathered}
v_{p}^{0}=v_{p}^{s} \frac{\left(R+1-R v_{2(1)}-v_{2(2)}\right)}{R+1} \\
V=\frac{(R+1)\left(V_{1}^{0}+V_{3}^{0}\right)}{R+1-R v_{2(1)}-v_{2(2)}}
\end{gathered}
$$

Substituting eq C-4 into C-1a and b, we obtain:

$$
\begin{aligned}
& V_{(1)}=\frac{R\left(V_{1}^{0}+V_{3}^{0}\right)}{R+1-R v_{2(1)}-v_{2(2)}} \\
& V_{(2)}=\frac{V_{1}^{0}+V_{3}^{0}}{R+1-R v_{2(1)}-v_{2(2)}}
\end{aligned}
$$

As $V_{1}^{0}$ and $V_{3}^{0}$ are initial conditions, $V_{2}, v_{p}^{0}$, and $V$ (also $V_{(1)}$ and $V_{(2)}$ are obviously functions of three parameters, $R, v_{2(1)}$, and $v_{2(2)}$. By use of eq C-la and b, $v_{X_{i}(1)}$ and $v_{X_{i}(2)}$ are expressed by $\mathrm{C}-6 \mathrm{a}$ and $\mathrm{b}$, respectively.

$$
\begin{aligned}
& v_{X_{i}(1)}=\frac{V_{3}^{0} g_{(1)}\left(X_{i}\right)}{V_{(1)}}=v_{p}^{0} \frac{R+1}{R} g_{(1)}\left(X_{i}\right) \\
& v_{X_{i}(2)}=\frac{V_{3}^{0} g_{(2)}\left(X_{i}\right)}{V_{(2)}}=v_{p}^{0}(R+1) g_{(2)}\left(X_{i}\right)
\end{aligned}
$$


Combining eq 27, C-3, C-6a, and b, eq 35a and $\mathrm{b}$ are derived. $v_{X_{i}(1)}$ and $v_{X_{i}(2)}$ are rewritten as follows,

$$
\begin{gathered}
v_{X_{i}(1)}=v_{p}^{0} \frac{R+1}{R+\exp \left(\sigma X_{i}\right)} g_{0}\left(X_{i}\right) \\
v_{X_{i}(2)}=v_{p}^{0} \frac{(R+1) \exp \left(\sigma X_{i}\right)}{R+\exp \left(\sigma X_{i}\right)} g_{0}\left(X_{i}\right)
\end{gathered}
$$

Utilizing eq C-6 and 34, eq C-8a and b are obtained.

$$
\begin{aligned}
& v_{p(1)}=v_{p}^{0} \frac{R+1}{R} \sum_{i=1}^{m} g_{(1)}\left(X_{i}\right)=v_{p}^{0} \frac{R+1}{R} \rho_{s}(\mathrm{C}-8 \mathrm{a}) \\
& v_{p(2)}=v_{p}^{0}(R+1) \sum_{i=1}^{m} g_{(2)}\left(X_{i}\right)=v_{p}^{0}(R+1) \rho_{p}(\mathrm{C}-8 \mathrm{~b})
\end{aligned}
$$

Substitution of eq C-3 into eq C-8a and b yields eq $36 \mathrm{a}$ and $\mathrm{b}$. Combination of $X_{n(1)}=$ $\sum g_{(1)}\left(X_{i}\right) /\left(\sum g_{(1)}\left(X_{i}\right) / X_{i}\right)$ and eq $33,34 \mathrm{a}$, and b gives following relation (eq 37 ):

$$
\begin{aligned}
\frac{1}{X_{n(1)}} & =\frac{1}{\rho_{s}}\left\{\sum_{i=1}^{m} \frac{g_{0}\left(X_{i}\right)}{X_{i}}-\sum_{i=1}^{m} \frac{g_{(2)}\left(X_{i}\right)}{X_{i}}\right\} \\
& =\frac{1}{\rho_{s}}\left\{\frac{1}{X_{n}^{0}}-\frac{\rho_{p}}{X_{n(2)}}\right\}
\end{aligned}
$$

In the same way, $1 / X_{n(2)}$ is represented by eq $\mathrm{C}-10$.

$$
\frac{1}{X_{n(2)}}=\frac{1}{\rho_{p}}\left\{\frac{1}{X_{n}^{0}}-\frac{\rho_{s}}{X_{n(1)}}\right\}
$$

\section{APPENDIX D}

\section{Derivation of eq 52 and 53}

At $v_{p}^{0}=$ constant and $\rho_{p}=1, V_{1}^{0}=0, V_{2}=$ $V-V_{3}^{0},\left(v_{1(1)}^{*}, v_{2(1)}^{*}, v_{p(1)}^{*}\right)=(0,1,0),\left(v_{1(2)}^{*}, v_{2(2)}^{*}\right.$, $\left.v_{p(2)}^{*}\right)=\left(0, \quad 1-v_{p(2)}^{*}, \quad v_{p(2)}^{*}\right), \quad g_{(1)}\left(X_{i}\right)=0, \quad$ and $g_{(2)}\left(X_{i}\right)=g_{0}\left(X_{i}\right)$ hold, and eq 30 becomes:

$$
\begin{gathered}
\ln \left(1-v_{p(2)}^{*}\right)+v_{p(2)}^{*}+\left(\frac{v_{p(1)}}{X_{n(1)}}\right)_{\rho_{p} \rightarrow 1}-\frac{v_{p(2)}^{*}}{X_{n}^{0}} \\
+\chi_{23}^{0}\left[v_{p(2)}^{* 2}+\sum_{r=1}^{n_{r}} p_{23, r} v_{p(2)}^{* r+2}\right]=0
\end{gathered}
$$

By use of eq C-8a and 9,

$$
\left(\frac{v_{p(1)}}{X_{n(1)}}\right)_{\rho_{p} \rightarrow 1}=\lim _{\rho_{p} \rightarrow 1}\left[v_{p}^{0} \frac{R+1}{R}-\frac{1}{X_{n(2)}}\right]=0
$$

Substitution of eq D-2 into D-1 yields eq 52 . Utilizing eq C-4, eq D-3 is obtained,

$$
V=\frac{\left(R^{*}+1\right) V_{3}^{0}}{v_{p(2)}^{*}}
$$

and eq D-3 is rewritten as eq 53 . Combining eq $\mathrm{C}-1 \mathrm{a}, \mathrm{b}$, and 53 , we can express $V_{(1)}^{*}$ and $V_{(2)}^{*}$ is expressed by eq D-4a and $b$, respectively.

$$
\begin{aligned}
& V_{(1)}^{*}=\left(1-\frac{v_{p}^{0}}{v_{p(2)}^{*}}\right) V \\
& V_{(2)}^{*}=\frac{v_{p}^{0}}{v_{p(2)}^{*}} V
\end{aligned}
$$

If we set solvent 1 as non-solvent and solvent 2 as good solvent, eq 52 becomes:

$$
\begin{aligned}
& \ln \left(1-v_{p(2)}^{*}\right)+v_{p(2)}^{*}-\frac{v_{p(2)}^{*}}{X_{n}^{0}} \\
& \quad+\chi_{13}^{0}\left[v_{p(2)}^{* 2}+\sum_{q=1}^{n_{q}} p_{13, q} v_{p(2)}^{* q+2}\right]=0
\end{aligned}
$$

\section{REFERENCES}

1. P. J. Flory, J. Chem. Phys., 12, 425 (1944).

2. R. L. Scott, J. Chem. Phys., 13, 178 (1945).

3. R. L. Scott, J. Chem. Phys., 17, 2689 (1949).

4. H. Tompa, Trans. Faraday Soc., 45, 1142 (1949).

5. M. Nakagaki and H. Sunada, Yakugaku Zasshi, 83, 1147 (1963).

6. W. R. Krigbaum and D. K. Carpenter, J. Polym. Sci., 14, 241 (1954).

7. K. W. Suh and D. W. Liou, J. Polym. Sci., A-2, 6, 813 (1968).

8. P. J. Flory, "Principle of Polymer Chemistry," Cornell University Press, Ithaca, New York, 1953.

9. K. Kamide, S. Matsuda, and Y. Miyazaki, Polym. J., 16, 479 (1984).

10. K. Kamide and S. Matsuda, Polym. J., 16, 515 (1984).

11. K. Kamide and S. Matsuda, Polym. J., 16, 591 (1984).

12. T. M. Aminavhavi and P. Munk, Macromolecules, 12, 607 (1979).

13. F. W. Altena and C. A. Smolders, Macromolecules, 15, 1941 (1982). 
Phase Equilibria of Quasi-Ternary Systems V.

14. K. Kamide, S. Matsuda, T. Dobashi, and M. Kaneko, Polym. J., 16, 839 (1984).

15. M. Kurata, "Thermodynamics of Polymer Solutions," Harwood Academic Publishers, Chur, London, New York, 1982, p 125, Chapter 2.

16. R. Koningsveld, L. A. Kleintjens, and A. R. Shultz, J. Polym. Sci., A-2, 8, 1261 (1970).

17. R. Koningsveld and L. A. Kleintjens, Macromolecules, 4, 637 (1971).

18. K. Kamide, Y. Miyazaki, and K. Sugamiya, Makromol. Chem., 173, 113 (1973).

19. K. Kamide and S. Matsuda, Polym. J., 18, 347 (1986).

20. see, for example K. Kamide, in "Fractionation of Synthetic Polymers," L. H. Tung, Ed., Marcel
Dekker Inc., New York, N. Y., 1977, Chapter 2.

21. K. Kamide, Y. Miyazaki, and T. Abe, Polym. J., 9, 395 (1977).

22. I. Noda, H. Ishizawa, Y. Miyazaki, and K. Kamide, Polym. J., 12,ه87 (1980).

23. K. Kamide, K. Sugamiya, T. Kawai, and Y. Miyazaki, Polym. J., 12, 67 (1980).

24. K. Kamide and Y. Miyazaki, Polym. J., 12, 205 (1980).

25. K. Kamide and Y. Miyazaki, Polym. J., 13, 325 (1981).

26. K. Kamide, Y. Miyazaki, and T. Abe, Br. Polym. J., 13, 168 (1981).

27. K. Kamide, T. Abe, and Y. Miyazaki, Polym. J., 14, 355 (1982). 